\title{
Hoplostethus islandicus, nov. spec. (Acanthopterygia, Abt. Beryciformes, Familie Trachichthyidae) aus den südisländischen Gewässern
}

\author{
Von Adolf Kotthaus, Bremerhaven \\ Aus der Biologischen Anstalt Helgoland, Abt. für Fischereiforschung, \\ Forschungsinstitut der Bundesanstalt für Fischerei \\ (Mit 2 Tabellen und 26 Abbildungen im Text)
}

In h a lt $\mathrm{s}$ ü bersicht: A. Fundgeschichte, S. 62 - B. Systematik und Verbreitung der Fam. Trachichthyidae, S. 63 - C. Morphologie und Anatomie von Hoplostethus islandicus, S. 66 - 1. Körperformen und -proportionen, S. 66 - 2. Beschuppung, S. 69 - 3. Der Kopf, S. 71 - 4. Die Flossen, S. 75 - 5. Das Skelett, S. 76 - 6. Innere Organisation, S. 81 7. Fettgehalt, S. $84-$ D. Zusammenfassung, S. 85 .

\section{A. Fundgeschichte}

Im November 1949 brachte der Bremerhavener Fischdampfer „Kap Kanin" von einer Reise nach den südisländischen Fanggründen fünf Fische mit, die bis dahin auf deutschen Fischmärkten nicht beobachtet wurden. Der 1. Steuermann des Dampfers, Herr Wennhoefer, überbrachte sie der Biologischen Anstalt Helgoland, Abt. für Fischereiforschung in Bremerhaven, als „absonderliche Rotbarschart". Außer einer gewissen Ähnlichkeit in Form und Größe und einer allerdings sehr weitgehenden Obereinstimmung in der Färbung, waren keine näheren Beziehungen zu Sebastes marinus festzustellen.

Die Tiere wurden zwischen dem 11. und 18. Nov. 1949 etwa $35 \mathrm{Sm}$ südlich von Ingolfshöfdi an der steil abfallenden Schelfkante des Oeraefagrundes in etwa $180 \mathrm{~m}$ Wassertiefe, und $z$ war alle fünf Exemplare im gleichen "Hol“, erbeutet. Der Grund war an dieser Stelle sehr "unrein", d.h. mit Korallen (Lophohelia) und Seepodken (Balanus hammerï) bestanden. Diese Gründe werden normalerweise wegen der Zerreißgefahr für die Netze von den Fischern gemieden, und es ist nur einer Unachtsamkeit in der Navigation zuzuschreiben, daß das Schiff vom rechten Kurs abkam und in diese "gefährliche" Region geriet.

Trotz eifriger Nachforschungen konnten lange Zeit keine weiteren Exemplare gefunden werden. Zwar sagten die Fischer übereinstimmend, daß sie solche Fische bereits seit etwa 20 Jahren gelegentlich unter Island gefangen, aber meist als nutzlosen Beifang wieder über Bord geworfen hätten. Auch ein durchaus glaubwürdiger Bremerhavener Filetiermeister gibt an, daß er schon vor vielen Jahren bisweilen solche Fische in den Rotbarschanlandungen aus isländischen Gewässern gefunden, aber nicht weiter beachtet habe.

Erst im Januar 1951 gelangten weitere Exemplare in unseren Besitz, und zwar wurden von dem Fischdampfer "Antares“, Kapitän BOSIEN, am 15. Januar 1951 an der südlichen Steilkante des, von den Fischern als "Schonersbank" bezeichneten, westlichen Teiles der Medallandbucht in $220-240 \mathrm{~m}$ Wassertiefe in vier aufeinanderfolgenden "Hols" 23 Exemplare gefangen. Der Grund bestand hier aus hellem Schlick. Wenige Tage später gerieten dem Fischdampfer „Spica“, Kapitän ABSHAGEN, etwas weiter östlich, am Steilabfall des 
Skaftatiefs ebenfalls in 220-240 m Wassertiefe weitere 8 Fische dieser Art ins Netz. Der Grund war an dieser Stelle "scharf", d. h. wieder mit Lophohelia und Balanus bestanden. Seitdem sind keine weiteren Funde mehr bekannt geworden ${ }^{*}$ ).

Bei diesen Fischen handelt es sich ausschließlich um adulte Formen von 54 bis $68 \mathrm{~cm}$ Totallänge und einem Gewicht von durchschnittlich $4 \mathrm{~kg}$. Der Erhaltungszustand ließ im allgemeinen sehr zu wünschen übrig, vor allem waren die sehr spröden Flossenstrahlen vielfach abgebrochen und mehrere Tiere wiesen Beschädigungen an Schuppenkleid und Flossen auf, was auf wenig sorgsame Behandlung beim Fang, an Bord und beim Sortieren auf dem Fischmarkt zurückzuführen ist. Offenbar sind es sehr alte Tiere, wie die große Ringzahl auf den Hartgebilden, die allerdings nicht eindeutig zu lesen sind, beweist. Außerdem sprechen dafür häufig auftretende Verkrüppelungen und Verwachsungen an den Flossenstrahlen, die in der Regel nur bei sehr alten Fischen angetroffen werden. Trotz dieser Mängel war das Material für die systematische Bearbeitung voll verwendungsfähig.

Ahnlich wie bei Sebastes marinus verblaßten die Tiere innerhalb kurzer Zeit in Formalin vollkommen zu einem schmutigen Weiß, so daß schon bald von der leuchtend roten Farbe, die gleichmäßig den ganzen Körper, einschließlich Flossen und Bauchseite, überzieht, nichts mehr zu erkennen war. Nur einige wenige Exemplare, die in Glycerin-Arsenik konserviert werden konnten, behielten ihre Farbe weitgehend bei.

\section{B. Systematik und Verbreitung der Familie Trachichthyidae}

Folgen wir der neueren systematischen Einteilung (WEBER \& BEAUfORT, JOUBIN, SMITH u. a.), so erweisen sich unsere Fische als zur Gattung Hoplostethus, Cuv. \& Val., Familie Trachichthyidae, Beeker, Ordnung Berycimorphi, Weber \& Beaufort, Ordnung Berycomorphoidea, Schultz \& Stern oder Abteilung Beryciformes, Joubin, Ehrenbaum, Smith, gehörig.

Zur Familie der Trachichthyidae werden nach der neueren Systematik die beiden Gattungen Trachichthys und Hoplostethus gezählt. WEBER \& BEAUFORT fügen hier als dritte Gattung noch Leiogaster mit einer Art an, die aber als identisch mit Hoplostethus melanopus erkannt worden ist.

Diese beiden Trachichthyidae-Gattungen unterscheiden sich durch recht wesentliche Merkmale eindeutig voneinander, und zwar zunächst in der Bezahnung; bei Hoplostethus sind Praemaxillare, Dentale und Palatinum bezahnt, bei Trachichthys zusätzlich auch der Vomer. Bei Trachichthys weichen die Schuppen der Seitenlinie in ihrer Größe kaum merklich von derjenigen der übrigen Körperschuppen ab, während sie bei Hoplostethus um ein Mehrfaches größer sind. Weitere Unterschiede beziehen sich auf die Zahl der Hartstrahlen in der Dorsalflosse, die Beschuppung des Kopfes, die Ausbildung des Infraorbitalringes u. a. m.

Auf Grund dieser Charakteristika gehören unsere Fische eindeutig zur Gattung Hoplostethus, so daß wir uns weiterhin auch nur mit dieser zu befassen brauchen. Von dieser Gattung sind bisher folgende zwölf Arten bekannt geworden: 1. H. mediterraneus (Cuv. \& Val., 1829); 2. H. japonicus (Hilgendorf, 1879); 3. H. atlanticus (Collett, 1889); 4. H. pacificus (German, 1899); 5. H. mediterraneus, var. late (Mc Culloch, 1914); 6. H. intermedius (Mc Culloch, 1914); 7. H. gigas (Mc Culloch, 1914); 8. H. elongatus (Griffin, 1928); 9. H. gilchristi (Smith, 1935); 10. H. melanopterus (Fowler, 1938); 11. H. (Aulohoplostethus) metallicus (Fowler, 1938); 12. H. melanopus (Weber, 1913) ${ }^{\text {s*t }}$ ).

Von vorgenannten zwölf Arten, worunter sich möglicherweise auch noch Synonyme befinden, sind bislang nur zwei aus den atlantischen Gewässern bekannt geworden, und zwar

*) Freundlicherweise stellte Herr Loges, Direktor der Fischdampferreederei „Nordstern", den größten Teil der Tiere der Biologischen Anstalt zur weiteren Bearbeitung zur Verfügung, wofür ihm auch an dieser Stelle gedankt sei.

**) Diese Liste darf wohl als vollständig angesehen werden; sie wurde mir freundlicherweise von Herrn Dr. TUCKER vom British Museum (Natural History) London mitgeteilt, wofür ich ihm neben anderen bereitwilligen Auskünften auch an dieser Stelle meinen aufrichtigen Dank ausspreche. 
H. mediterraneus und H. atlanticus. Alle anderen stammen aus dem indo-pazifischen, japanischen oder australischen Raum.

Interessant ist die Tatsache, daß bisher alle Funde von Hoplostethus-Arten sich auf die warmen und gemäßigten Breiten bis etwa zum $51 .^{\circ} \mathrm{N}$ bzw. $45 .^{\circ} \mathrm{S}$ beschränken, sowohl auf der westlichen, als auch auf der östlichen Hemisphäre, daß nun aber plötzlich eine Art in so hohen Breiten wie Island (zwischen 63 bis $64^{\circ} \mathrm{N}$ ) gefangen wurde.

$\mathrm{Zu}$ einer der beiden atlantischen Arten gehören unsere Fische zweifellos nicht, wie weiter unten ausführlich darzustellen sein wird. Ob sie mit Formen der östlichen Hemisphäre identisch sind, vermag im Augenblick nicht entschieden zu werden; denn es stand mir weder Vergleichsmaterial zur Verfügung, noch war mir alle, diese Arten betreffende Literatur zugänglich. Mir scheint aber diese Möglichkeit kaum in Betracht zu kommen, einmal wegen der weiten räumlichen Trennung ibres Vorkommens, zum anderen aber auch in Anbetracht der Tatsache, daß unsere Fische in erheblich viel nördlicheren Breiten gefangen wurden, als sich das Verbreitungsgebiet der anderen Arten erstreckt.

Ich befolge daher den Rat von Herrn Dr. TUCKER, unsere Fische als neue Art zu beschreiben, selbst auf die Gefahr hin; daß sich bei späterer Überprüfung die Identität mit einer der bereits beschriebenen Arten aus anderen Meeresteilen herausstellen sollte, und nenne die Tiere Hoplostethus islandicus (weiterhin abgekürzt $H$. isl.).

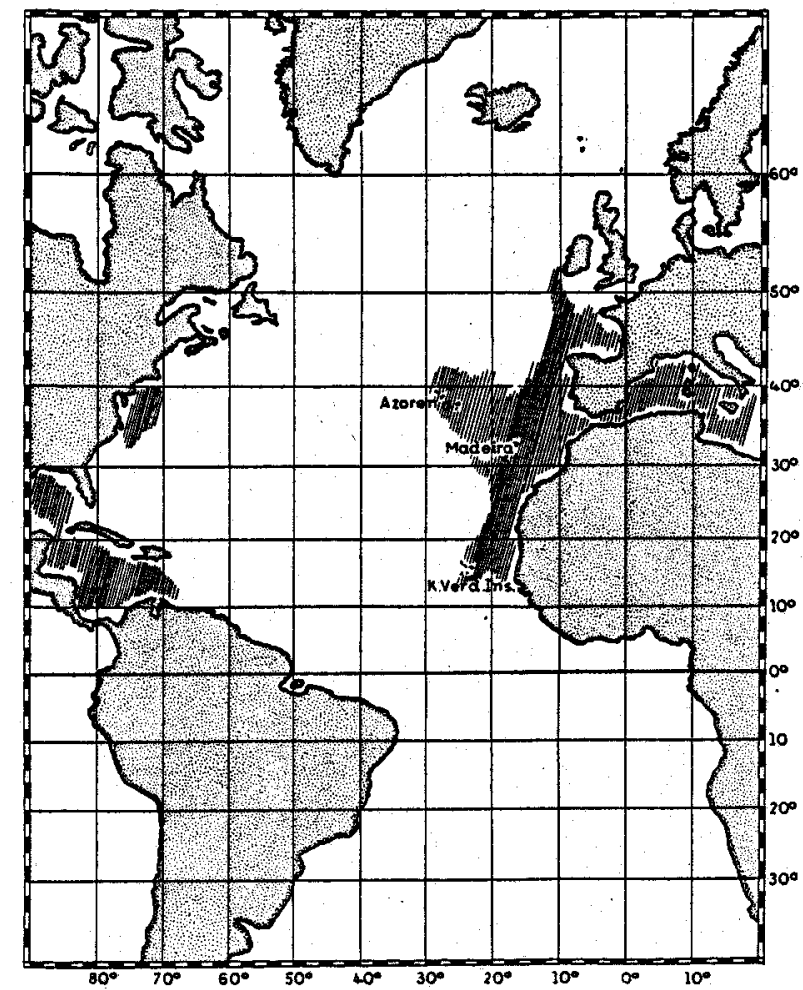

Abb. 1. Karte des Verbreitungsgebietes der atlantischen Hoplostethus-Arten (schräg schraffiert: $H$. mediterraneus; -waagerecht schraffiert: $H$. islandieus; O-Fundstelle von $H$. atlanticus.)

Während $H$. mediterraneus (weiterhin abgekürzt $H$. med.) gelegentlich der verschiedenen Tiefsee-Expeditionen ("Valdivia“, „Michael Sars", „Traveilleur", „Talisman“, „Hirondelle“, "Dana" u.a.m.) durch zahlreiche Funde bekannt geworden ist, wurden von $H$. atlantictus (weiterhin abgekürzt $H$, atl.) bisher erst zwei Exemplare in der Nähe von Flores (Azoren) gefunden. Nach S SITH soll diese Art allerdings auch im Tiefen des Kapgebietes vorkommen.

Auf Grund der Fangortangaben in den verschiedensten Expeditionsberichten kann man das Verbreitungsgebiet von $H$. med. in den atlantischen Gewässern und im Mittelmeer etwa so abgrenzen, wie es in Abb. 1 geschehen ist, d. h. es erstreckt sich auf den westlichen Teil des 
Mittelmeeres, einschließlich der südlichen Adria (SOLJAN), entlang den tieferen Küstengebieten des westlichen Atlantiks vom Sudan bis südwestlich Irland, einschließlich der Gewässer um die Kap Verden, Madeira und Azoren. Im westlichen Atlantik wurde die Art im Karibischen Meer. im Golf von Mexiko und entlang der Atlantikküste bis zum 40. ${ }^{\circ} \mathrm{N}$ festgestellt. Weitere Funde stammen aus dem Kapgebiet (SMITH), von der ostafrikanischen Küste (B AUER), aus dem Bengalischen Golf (ALCOCK, 1894) und aus den japanischen Gewässern (HILGENDORF). In all diesen Gebieten lebt H.med. bathypelagisch, und zwar bevorzugt er die Tiefen von 235 bis $780 \mathrm{~m}$; darüber und darunter wurden nur gelegentlich einige Tiere gefangen. Es sind also Fische, die auf die "Warmwassersphäre“ (WUEST) der Erde beschränkt sind. Nach den Literaturangaben ist zu schließen, daß $H$. med. eine größte Länge von etwa $30 \mathrm{~cm}$ erreicht. Es handelt sich also, wie bei den meisten Beryciformes, um eine verhältnismäßig kleinwüchsige Form. Größer dürfte $H$. atl. kaum werden, obgleich hierüber nur zwei Größenangaben vorliegen $(13,5 \mathrm{~cm}$, Colletr und $23,8 \mathrm{~cm}$, Rés. Camp. du Prince de Monaco). $H$. isl. ist dagegen mit Längen von $54-68 \mathrm{~cm}$ schon als Riesenform zu bezeichnen.

Es erhebt sich die Frage, wie es möglich ist, daß eine so große Fischart in einem Gebiet, das sowohl durch die Fischerei als auch durch meereswissenschaftliche Untersuchungen als wohlerforscht angesehen werden kann, bisher noch unentdeckt geblieben ist. Es gibt dafür verschiedene Deutungsmöglichkeiten, die aber nicht alle restlos befriedigen können. Daß $H$. isl. nicht erst seit 1949 unter Island zu fangen war, geht aus den übereinstimmenden Aussagen unserer Fischer hervor. Zumindest sollen die Tiere seit etwa 20 Jahren in den Fängen aufgetreten sein, also etwa ab 1930. Das ist aber ein Zeitpunkt, der in etwa mit dem Beginn der für die nordatlantischen Gebiete festgestellten Erwärmung zusammenfällt. Diese Erwärmung hatte auch in biologischer Hinsicht mancherlei Folgen; vor allem konnte festgestellt werden, daß seit dieser Zeit eine Anzahl von Seetieren ihr bisheriges Verbreitungsgebiet sehr erheblich nach Norden ausdehnten (FrIDRIKSSON), andere, die bisher nur aus südlicheren Breiten bekannt waren, wurden nun auch gelegentlich unter Island, meist aber schon früher an der Südnorwegenküste festgestellt. Man könnte nun geneigt sein, auch für unsere Tiere eine Ausdehnung des Verbreitungsgebietes anzunehmen, zumal ihre nächsten Verwandten, $H$. med. u. $H$. atl., Formen der "Warmwassersphäre" sind. Gegen diese Auffassung spricht vor allem, $\mathrm{daB} H$. isl. aus südlicheren Breiten nicht bekannt geworden ist und daß er bisher auch noch nicht an der südnorwegischen Küste angetroffen wurde (private Mitteilung von Dr. E. KOEFOED-Bergen). Außerdem gibt noch 'folgende Tatsache zu denken: H. isl. ist aller Wahrscheinlichkeit nach kein pelagisch bzw. bathypelagisch lebender Fisch wie $H$. med und $H$. atl., die über eine wohlausgebildete Schwimmblase verfügen, sondern ein Boden$\mathrm{f}$ i $\mathrm{s} \mathrm{ch}$, dessen Schwimmblase nicht mehr oder nur in ganz geringem Maße funktionsfähig ist (s. S. 83). Für einen Bodenfisch aber besteht wenig Wahrscheinlichkeit, daß er aus südlichen Breiten bis nach Island vordringt. Auf der anderen Seite würde bei einer bathypelagischen Lebensweise eine Erwärmung, die nur die oberflächlichen Wasserschichten bis etwa 200/300 m betrifft, keinen Einfluß auf die Ausdehnung des Verbreitungsgebietes haben können.

Auch die Möglichkeit einer Verfrachtung durch besondere Strömungsverhältnisse könnte man in Betracht ziehen, aber auch hierfür ist die Wahrscheinlichkeit gering, wenngleich auch bei einem Bodenfisch ein Einfluß der Strömung durchaus nicht ganz von der Hand zu weisen ist.

Schließlich, und das wäre freilich die nächstliegende Vermutung, könnte man annehmen, daß $H$. isl. unter Island endemis $\mathrm{ch}$ ist, aber nur auf Grund seiner besonderen Lebensweise bisher nicht gefunden wurde. Alle Fische wurden am Steilhang des südisländischen Schelfes gefangen, wo der Abfall so schroff ist (auf wenige hundert Meter ein Abfall von $700-1000 \mathrm{~m})$, daß hier in der Regel nicht gefischt werden kann. Zudem ist dieser Hang ein beliebter Ansiedlungspunkt für Korallen und Seepodken, die ohnehin eine Fischerei so gut wie ausschließen. In allen drei Fällen, wo $H$. isl. gefangen wurde, waren die Dampfer zufällig vom eigentlichen, dicht dabeiliegenden, reinen" Fischgrund abgekommen. Wenn wir also annehmen, daß dieser unbefischbare. Steilhang des isländischen Schelfes die eigentliche Heimat unserer Fische ist, dann ist es verständlich, daß sie normalerweise unbehelligt durch die Fischerei leben und nur durch Zufall ins Netz geraten können. Ein Umstand scheint mir auch noch dafür zu sprechen, daß die Tiere in ihrem eigentlichen Verbreitungsgebiet gefangen wurden; nämlich der Reifezustand der Gonaden bei den Exemplaren vom Januar 1951 ließ auf eine dicht bevorstehende Laichzeit schließen (Reifestadium V). Wir wissen aber von den bekannteren Fischen, daß sie sich im allgemeinen auf diesem Stadium bereits auf ihren Laichplätzen befinden, zumindest keine größeren Wanderungen mehr ausführen.

So bestechend vielleicht auf den ersten Blick diese simple Erklärung scheint, so lassen sich doch auch Gegenargumente anführen, auf die mich Herr Dr. BERTELSEN-Charlottenlund 
freundlichst hinwies. Das ist nämlich die Tatsache, daß in dem sehr umfangreichen dänischen Planktonmaterial aus diesem Meeresgebiet bisher weder Eier noch Larven berycider Fische gefunden wurden. Dieser Einwand ist aber nicht unbedingt stichhaltig, worauf auch BE?TELSEN selbst hinweist, wenn nämlich $H$. isl. sowohl bathypelagische Eier als auch Larven hätte oder lebendgebärend wäre und bathypelagisch lebende Larven besäße. Ob dem aber so ist, dafür lassen sich bisher noch keine Anhaltspunkte finden. Wir müssen daher einstweilen die Frage nach der wirklichen Heimat dieser überaus interessanten Fische offen lassen.

Das glücklicherweise in so reicher Zahl vorhandene Material gibt uns Gelegenheit, die Morphologie und Anatomie dieser Fische über das zur Artcharakteristik notwendige Maß hinaus zu studieren. Im Folgenden soll zunächst die Artbeschreibung im Vordergrunde stehen. Leider stand mir zu meinen jetzigen Untersuchungen kein Vergleichsmaterial von $H$. med. oder $H$. atl. zur Verfügung, so daß ich lediglith zum Vergleich die Beschreibungen aus der Literatur heranziehen konnte. Aber die vorhandenen wenigen Angaben genügen durchaus, klarzustellen, daß unsere Fische keineswegs mit einer der beiden anderen Hoplostethus-Arten des Atlantiks identisch sind*).

\section{Morphologie und Anatomie von Hoplostethus islandicus, n. spec.}

1. Körperformen und - proportionen.

Die im ersten kurzen Bericht (Kotrhaus) gebrachte Aufnahme läßt nur schwerlich das Tier in seiner wirklichen Gestalt und in seinen Formen erkennen; jedenfalls treten darin Einzelheiten, die für die Artcharakteristik wesentlich sind, nicht deutlich hervor. Vor allem sind die Skulpturierung des Kopfes und die Form der Flossen, weil verstümmelt, nicht zu erkennen. Einen wirklichen Eindruck von den Fischen bekommen wir erst an Hand der Zeichnung (Abb. 2), die in allen ihren Teilen als maßstabgetreu und formrichtig anzusehen ist, wenngleich manche Konturen am Tier selbst nicht so scharf hervortreten. Zur besseren Kenntlichmachung wurden die Stachelstrahlen der Flossen etwas dunkler als die Weichstrahlen gehalten. Die Flossenform wurde durch Heranziehen einer ganzen Reihe von Individuen rekonstruiert, da kein Tier alle Flossen komplett aufwies.

Wenn wir von der Caudalen absehen, so können wir die Gestalt von $H$. isl. am treffendsten als tropfenförmig bezeichnen. Bei geschlossenem Maul bildet das Kopfprofil nahezu einen Halbkreis. Jedenfalls fehlt ihm der für $H$. med. charakteristische Buckel am oberen Kopfprofil. In seiner Gestalt gleicht $H$. isl. wesentlich mehr dem Trachichthys Darwinii (Johnson) als $H$. med., wird aber durch seine weiteren Merkmale einwandfrei als zur Gattung Hoplostethus gehörig gekennzeichnet.

Die Rückenkontur ist durchgehend vom Schädel bis zum Ende der D gleichmäßig konvex gekrümmt, während sich das Bauchprofil, je nach dem Entwicklungsgrad der Gonaden, bald schwach, bald stark konvex ge-

*) Die erste und beste Beschreibung von H. med. finden wir bei Cuvier \& VALENCIENNES von 1829; Beschreibungen späterer Autoren (GONTHER, MOREAU, GOODE \& BEAN usw.) stützen sich in der Hauptsache auf diese Autoren und können nur als Bestätigung der Richtigkeit dieser Beschreibung angesehen werden. Nur V AILLANT bringt neue interessante Einzelheiten. Alle diese Arbeiten beziehen sich in der Hauptsache auf die äußere Beschreibung der Tiere, während die Anatomie recht kurz dabei wegkommt. Von F. SupiNo liegt eine Arbeit über das Schädelskelet von $H$. med. vor, die für einen Vergleich mit $H$. isl. durchaus hinreichend ist. SUPINO $s$ Zeichnungen zu dieser Schrift, die mir nur in der Reproduktion einer Mikroaufnahme zur Verfügung standen, lassen allerdings viel an Deutlichkeit zu wünschen übrig, zumal im Wesentlichen nicht einzelne Knochen, sondern nur das gesamte Cranium in verschiedenen Ansichten dargestellt wurden. - Für $H$. atl. liegen die Verhältnisse wesentlich ungünstiger, liegt hierüber doch nur die kurze Diagnose von COLIETT vor, die aber genügt, um erkennen zu lassen. daß $H$. atl. und $H$. isl. nicht ein und dieselbe Art sind. 


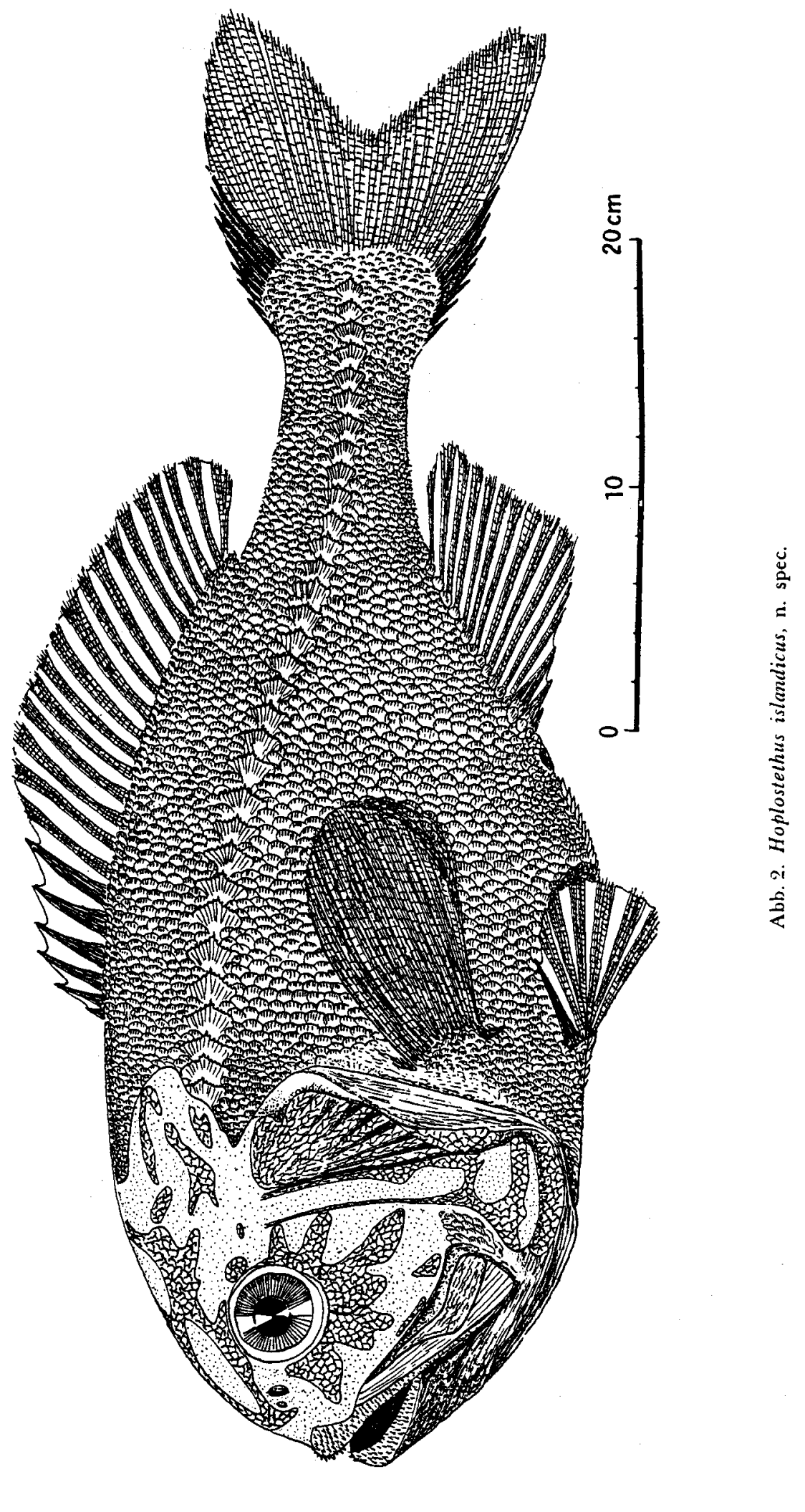


krümmt erweist. Der Schwanzteil verjüngt sich caudalwärts vom Ende der $\mathrm{D}$ und A nur wenig und ist zum Schluß breit ausladend als Flossenstrahlenträger für die $\mathrm{C}$ ausgebildet.

Ebenso wie bei den beiden anderen Arten ist der Körper von $H$. isl. seitlich stark kompreß, wirkt aber im ganzen gesehen wesentlich schlanker als bei jenen.

In ihren Körperproportionen weisen die drei hier zum Vergleich stehenden Arten recht erhebliche Unterschiede auf. Man könnte zunächst geneigt sein, sie mit den Größenunterschieden in Verbindung zu bringen; denn bekanntermaßen ändern sich die Körperproportionen im Laufe des individuellen Wachstums nicht unerheblich. Gerade in bezug auf verschiedene Berycidenarten haben LUETKEN und speziell für $H$. med. BraUER in dieser Hinsicht interessante Ergebnisse veröffentlicht. So wies LUETKEN z. B. nach, daß verschiedene Rhynchichthysarten nichts anderes als Jugendstadien von Holocentrum sind und daß die Jugendform vom Myripristis als Rhamphoberyx beschrieben wurde.

Da wir es aber mit Sicherheit bei $H$. med. und $H$. isl., wahrscheinlich auch bei $H$. atl., mit ausgewachsenen Tieren zu tun haben, deren Körperproportionen

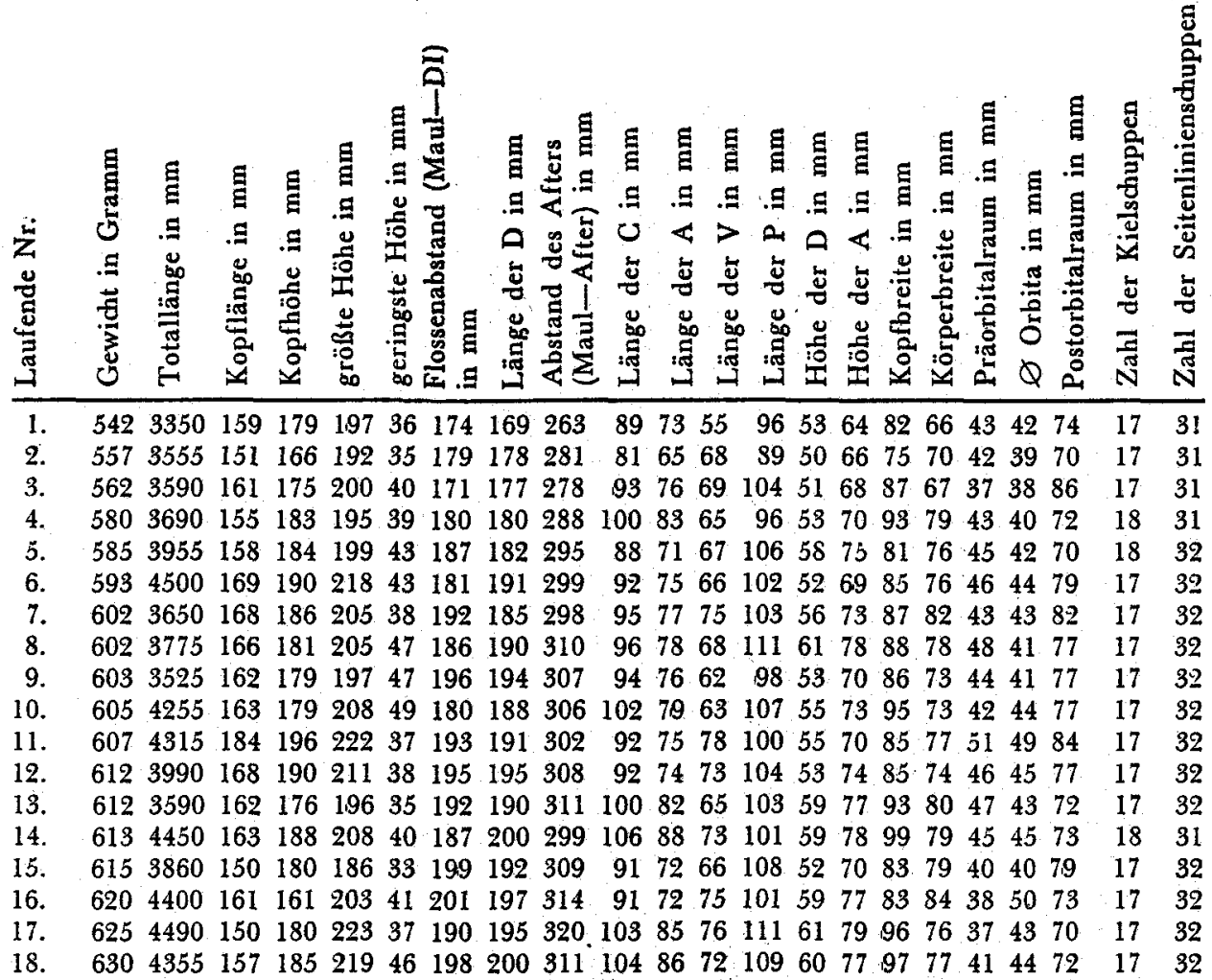

Dorsale: $3 \times \mathrm{VI} / 17 ; 6 \times \mathrm{VI} / 18$.

Caudale: $7 / 19 / 7 ; 7 / 20 / 6 ; 8 / 19 / 6 ; 7 / 19 / 7 ; 7 / 18 / 7 ; 7 / 19 / 6 ; 7 / 18 / 7$;

$8 / 21 / 6 ; 7 / 19 / 7 ; 7 / 20 / 6$.

Anale: $4 \times \mathrm{III} / 11 ; 7 \times \mathrm{III} / 12$

Ventrale: $11 \times \mathrm{I} / 6$.

Pectorale: $2 \times 18 ; 8 \times 19 ; 1 \times 20$

Tabelle 1.

Messungen an 18 Hoplostethus islandicus vom 15. Januar 1951. 
sich also kaum noch erheblich ändern dürften, so scheint es mir unbedenklich, in diesem Falle die relative Größe der Tiere unberücksichtigt zu lassen.

Zur Durchführung eines Vergleiches war es notwendig, die von den früheren Autoren angegebenen Maße, soweit sie vorliegen, in \% der Totallänge, oder bei Kopfmaßen als \% der Kopflänge umzurechnen*). In manchen Fällen mußten diese Werte aus den Abbildungen rekonstruiert werden. Die für $H$. isl. angegebenen Werte stellen die Mittel von Messungen an 18 Individuen dar, die im einzelnen in Tabelle 1 zusammengestellt sind.

In der Hauptsache wird sich unser Vergleich auf $H$. isl. und $H$. med. beschränken müssen; denn für $H$. atl. liegt nur die kurze Beschreibung von ColletT mit wenigen Maßangaben vor, und eine Abbildung des Tieres fehlt uns auch.

Der Ubersichtlichkeit halber sind die Körpermaße der drei Arten in Tabelle 2 einander gegenübergestellt. Es erübrigt sich daher, im einzelnen darauf einzugehen, nur soll noch einmal kurz auf die wesentlichsten Unterschiede hingewiesen werden.

Von den beiden anderen Arten ist $H$. isl. durch einen kleineren Kopf, niedrigeren Schwanzstiel, längere und niedrigere Dorsalflosse, kürzere Pectoralund Ventralflossen, sowie durch ein wesentlich kleineres Auge unterschieden.

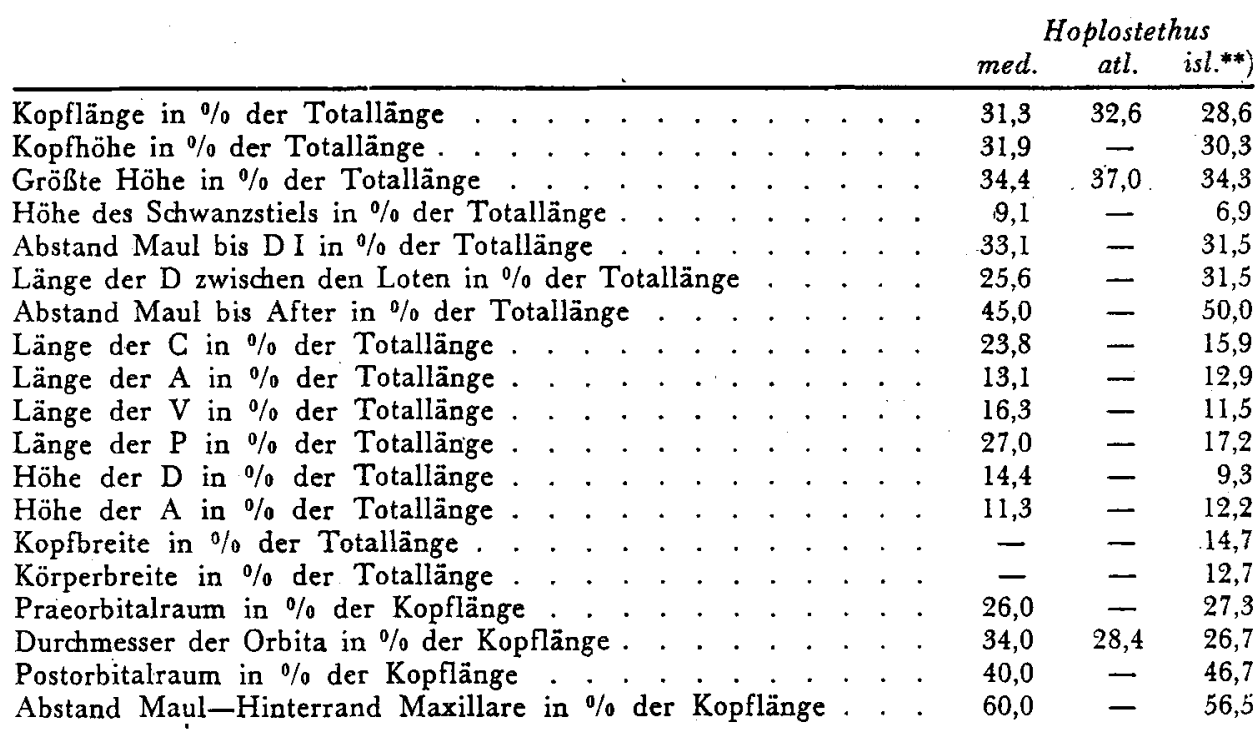

Tabelle 2.

Gegenüberstellung der Körpermaße der drei atlantischen Hoplostethusarten.

2. B e s chuppung.

Der Körper von $H$. isl. ist von unregelmäßig angeordneten, in Form und Größe außerordentlich stark variierenden Schuppen bedeckt. So finden wir in der Regel hochovale und große Schuppen an den Körperseiten (Abb. 3), kleinere rundliche oder trapezförmige an der Dorsal- und Ventralseite sowie auf dem Schwanzstiel (Abb. 4).

*) Ich folge hier der Methode, diese Prozentzahlen zu verwenden statt der bislang üblichen Angabe, wie oft ein Körpermaß in der Total- oder Kopflänge enthalten ist (vgl. BIGELOW, FARFANTE \& SCHRODER).

**) Jeweils Mittelwerte von 18 Messungen. 
Wir müssen diese Schuppen als ctenoid bezeichnen, wenngleich sie von der Form, die man sich im allgemeinen darunter vorstellt, wesentlich abweichen. Am ehesten könnte man sie als Mittelstück zwischen Cycloid- und Ctenoidschuppe ansprechen. Der distale, freie Teil der Schuppe ist mit kleinen, nahezu parallel verlaufenden Leisten in sehr wechselnder Zahl (etwa 10-20) besetzt, die jeweils in einen kleinen Dorn auslaufen. Im Gegensaty zu den noch zu besprechenden Kiel- und Seitenlinien-Schuppen sitzen die gewöhnlichen Körperschuppen sehr lose und waren demzufolge, vor allem bei den im Januar 1951 gefangenen Tieren, die nicht sehr sorgfältig behandelt worden waren, weitgehend aus ihren Taschen gelöst. Wegen der unregelmäßigen Anordnung läßt sich keine genaue Zahl der Schuppen einer Vertikalreihe angeben.

Bei den beiden anderen Arten sind die Körperschuppen entweder vollkommen glatt (vergl. VAILlant, Taf. 27, Abb. 5b) oder aber nur mit kleinen Dörnchen besetzt.

$\mathrm{Ki}$ el s chuppen. Zwischen dem Ansat der Ventralflossen und dem Anus bilden 17 bis 18 (nicht $30-32$, wie infolge eines Druckfehlers bei Kotrhaus 1950 steht) große, V-förmig gebogene Schuppen einen scharfen, gesägten Kiel, und zwar ausgeprägter als wir ihn z. B. beim Hering (Clupeaharengus) oder beim Sprott (Clupea sprattus) finden. Diese Schuppen sind in der Mitte der Kiellinie am größten. Ihre Flügel (Abb. 5) sind in den distalen, freien Teilen mit $4-5$ scharf hervortretenden Leisten besetzt, die z. T. in einem Dorn endigen; besonders tritt der mittlere breite und lange Stachel in Erscheinung.
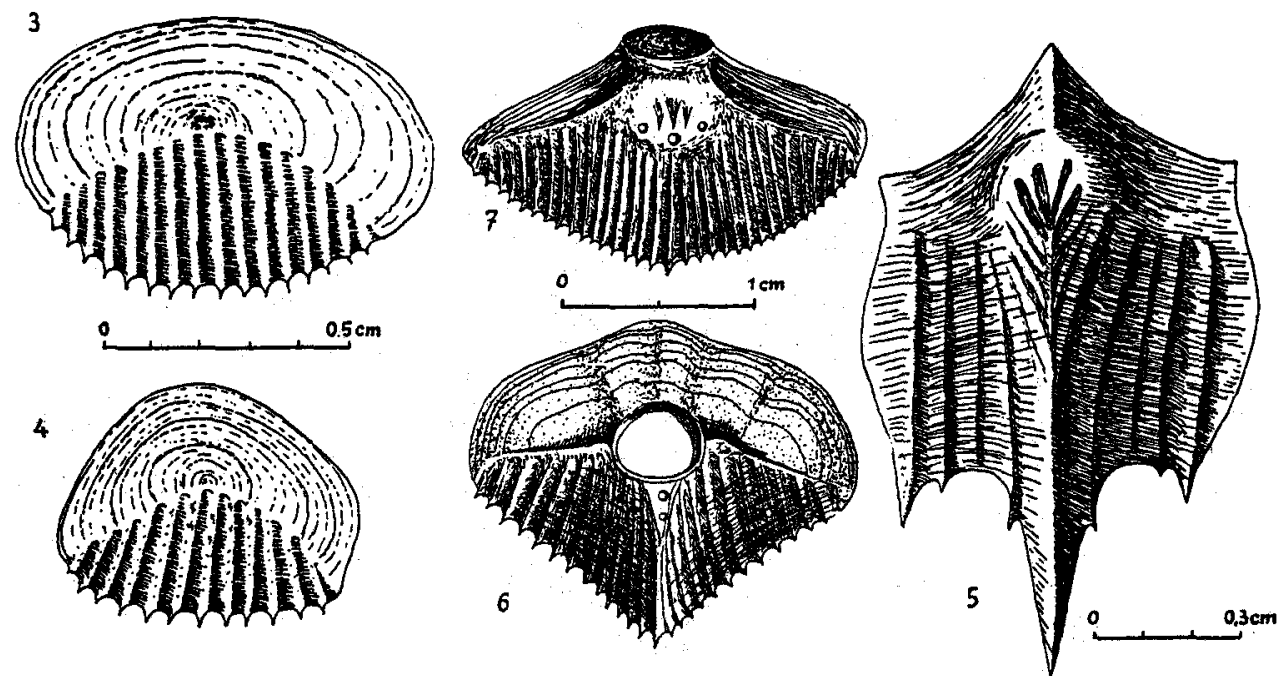

Abb. 3-7. Hoplostethus islandicus, n. spec., Schuppen: 3-4. Körperschuppen; 5. Kielschuppe; 6-7. Schuppen der Seitenlinie.

Bei $H$. med. finden wir ähnlich gestaltete Kielschuppen, aber in geringerer Zahl. Cuvier \& Valenciennes und Varllant geben 11, Lowe $11-13$ an; MOREAU dagegen teilt eine Variationsbreite von 8-13 mit. Bei H. atl. ist nach Colletr die Kiellinie sẹr undeutlich und schwach ausgebildet.

$S c h u p p e n d e r L$ in e 1 a teralis. Im Vergleich zu den normalen Körperschuppen sind die der Linea lateralis außerordentlich groß und von 
eigenartiger Gestalt. Ihre Form ist nicht im ganzen Bereich der Seitenlinie die gleiche. Als Beispiel mögen die Abbildungen 6 und 7 dienen, und zwar stammt erstere von einer Schuppe aus dem proximalen, die zweite aus dem distalen Abschnitt. Die Flügelbreite beträgt bei diesen Schuppen 20-21 mm, die Länge bei Abb. $616 \mathrm{~mm}$, bei Abb. 7 nur $12 \mathrm{~mm}$. Auffallend ist vor allem. daß der Basalteil der Schuppe röhrenartig den Seitenlinienkanal umgibt, daher sitzen diese Gebilde auch außerordentlich fest und sind kaum ohne Beschädigung herauszuziehen.

Der verdeckte, sehr dünne proximale Abschnitt nimmt, je weiter wir caudalwärts kommen, mehr und mehr an Breite ab. Auf ihm erkennen wir zahlreiche, eng beieinander liegende Ringe, die aber nicht eindeutig als Jahresringe $\mathrm{zu}$ lesen sind.

Der freie Teil der Schuppen, der in der Mitte etwas vorgewölbt ist und an dieser Stelle kleine Poren in verschiedener Zahl (2-6) besitgt, trägt, ähnlich wie die übrigen Körperschuppen, entweder nahezu parallel verlaufende oder caudalwärts etwas divergierende Leisten (ca. 20-30), die jeweils in einen kleinen scharfen Dorn auslaufen, von welchen der mittlere der stärkste ist. Bei den vorderen Schuppen ist der caudale Rand mehr winklig, bei den hinteren aber mehr abgerundet. Der zentrale, vorgewölbte Teil trägt zwischen den Poren noch 3-5 kleine, caudalwärts gerichtete Dornen.

Bei $H$. med. sind die Flügel der Schuppen sehr u n $\mathrm{l}$ e i ch m äß i g ausgebildet (vergl. Varllant, Taf. 27, Abb. 5c), d. h. der dorsale Flügel erscheint gegenüber dem ventralen wesentlich verlängert. Auch bei ihnen bilden die basalen Teile eine Röhre zur Aufnahme des Seitenlinienkanals, aber auf dem freien Teil finden wir nicht die charakteristischen Leisten, wie bei $H$. isl., sondern nur einen Besaty mit sehr kleinen, caudalwärts gerichteten Dörnchen.

Bei den isländischen Tieren schwankt die Zahl der Seitenlinien-Schuppen zwischen 31 und 32, bei $H$. med. beträgt sie 28-31. Für H. atl. ist keine Zahl verzeichnet.

Im Gegensaty zu H. med., dessen Kopf nackt sein soll, finden wir bei $H$. isl. an zwei engbegrenzten Stellen der Wangen und am Kinn Schuppen. An den Wangen, und zwar in dem Raum zwischen Praeoperculum, Infraorbitalring und Maxillare, siten jederseits etwa 30 Schuppen, von ähnlicher Gestalt wie die Körperschuppen, nur von geringerer Größe.

Ganz absonderlich gestaltete Schuppen treffen wir auf einem Wulst zwischen den beiden Unterkiefern an (Abb. 8 u. 9). Es sind kleine, etwa $4 \mathrm{~mm}$ lange und 2,5 mm breite Gebilde, die äußerst eng aneinander liegen und deren freie Teile zentral napfförmig vertieft sind. Die kleinen Höhlungen sind mit einem Sekret angefüllt und nach außen durch ein sehr dünnes Häutchen abgeschlossen. Der äußere horizontale Rand des Näpfchens ist mit einer großen Zahl kleiner, spitzer Dornen bestanden, so daß der Eindruck einer Bezahnung entsteht, ähnlich wie wir sie auf Praemaxillare und Dentale finden. Dem Baue nach darf man annehmen, daß es sich hier um Organe des Tastsinnes handelt.

\section{De $\mathrm{K}$ op $\mathrm{f}$.}

Wie bei allen Trachichthyidae-Arten, ist auch bei unseren Fischen der Kopf durch die charakteristische dermale Lage des Exoskelets ausgezeichnet, d. h. alle Deckknochen des Schädels einschl. der Opercularknochen liegen entweder mit der ganzen Oberfläche oder aber mit leistenartigen Knochenbrücken, nur vom Periost umgeben, frei zutage. Dabei kommt die außerordentlich 


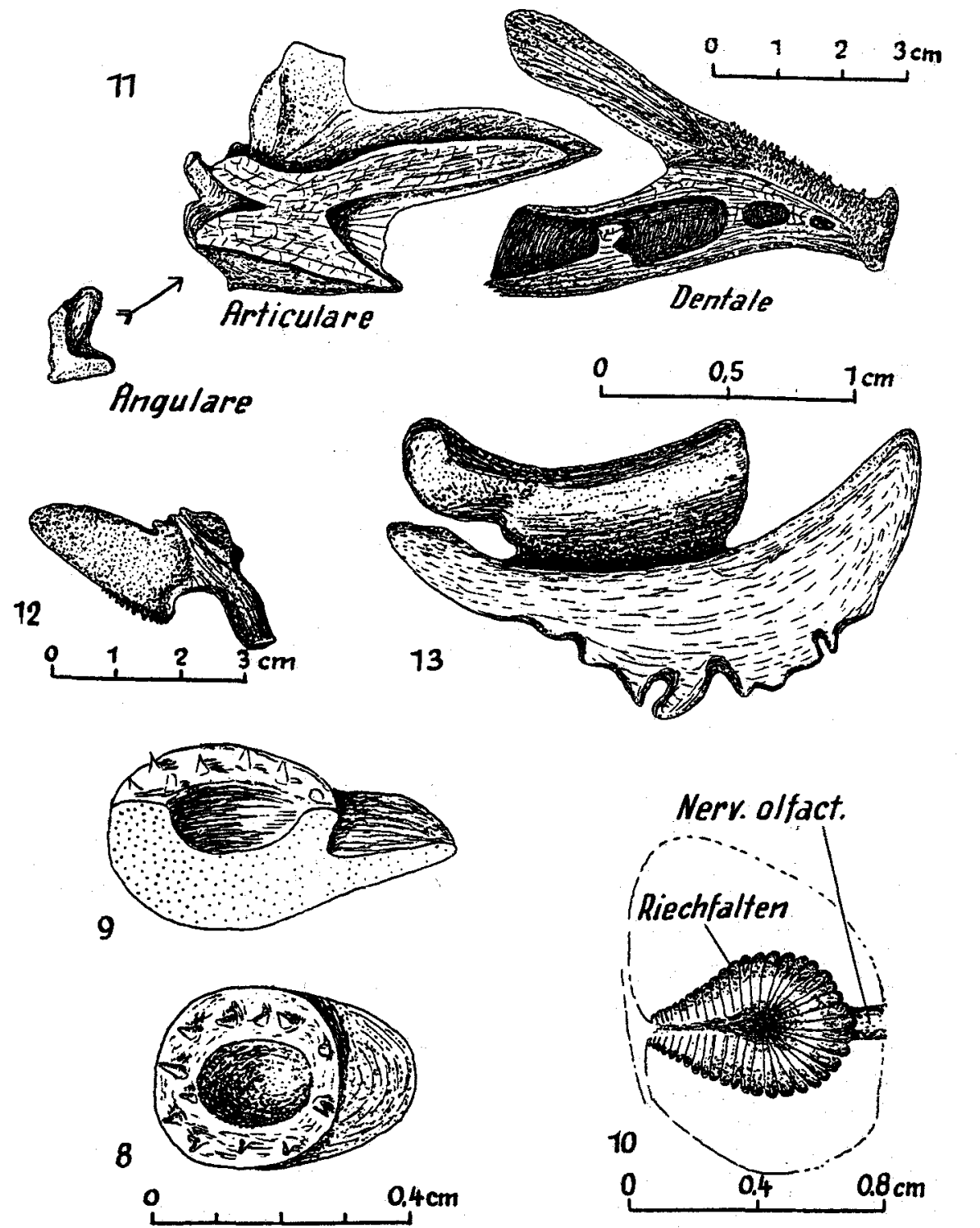

Abb. 8-13. Hoplostethus islandicus, n. spec.: 8. Schuppe vom Kinn, Aufsicht; 9. Schuppe vom Kinn, Längsschnitt; 10. linke Nasengrube; 11. Unterkiefer rechts; 12. Palatinum rechts; 13. Linker Statolith ("Sagitta“), Ansicht von innen.

lockere, schwammartige Struktur dieser Knochen sehr deutlich zum Vorschein. Alle freiliegenden Knochenteile sind daher sehr rauh, zumal auch die Ränder der einzelnen Lamellen stark gesägt bzw. bedornt, die inneren Flächen aber stets glatt und homogen sind.

Zwischen diesen Knochenbrücken dehnt sich ein weitverzweigtes und umfangreiches Schleimgefäß-System aus, das durch Lücken in den Knochenbrücken kommuniziert und unter dem Posttemporale (Suprascapulare älterer Autoren) mit dem Seitenliniensystem in Verbindung tritt. Nach außen sind diese Schleimhöhlen durch eine äußerst dünne, transparente und poröse Haut abgeschlossen. Insgesamt lassen sich drei Hauptgefäße unterscheiden, und zwar 
einen dorsalen, innerhalb des Frontale aufgespaltenen Ast, der vom Posttemporale durch das Postfrontale, Parietale, Frontale zum Praefrontale zieht und hier mit dem zweiten Ast, der innerhalb der Infraorbitalia verläuft, in Verbindung tritt. Dieser letgtere Ast zweigt sich im Petrosum vom dorsalen Zweig ab. Direkt dahinter, durch zwei hakenartig gebogene Fortsätze des Pteroticums, tritt der hintere Schleimkanal in das Praeoperculum ein und erstreckt sich über das Interoperculum, das Articulare bis zum Dentale.

Am Boden der Schleimhöhlen, namentlich in dem oberen Teil des Systems, finden wir zahlreiche, stecknadelkopfgroße Tuberkelchen, die möglicherweise Sinneshügel oder schleimsezernierende Drüsen darstellen. Der Erhaltungszustand unserer Tiere war leider nicht so, daß es noch möglich wäre, durch histologische Untersuchung klarzustellen, um welche Organe es sich in Wirklichkeit handelt.

$\mathrm{D}$ i e $\mathrm{N}$ a s e. Hoplostethus islandicus verfügt über ein wohlausgebildetes Geruchsorgan. Dieses liegt in einer geräumigen Höhle, die vorn und oben vom Praefrontale (Abb. 26, Prfr.), unten vom Infraorbitale I (Lacrymale) (Abb. 20) und medial vom Exethmoideum (Abb. 19) begrenzt wird. Zwischen Orbita und Geruchsgrube besteht nur eine häutige Trennungswand. Nach außen wird die Verbindung durch zwei hintereinander liegende Nasenöffnungen hergestellt, von denen die vordere kleinere einen größten Durchmesser von 6,5, die dahinter liegende größere einen solchen von $9,5 \mathrm{~mm}$ besitzt (Totallänge des Tieres $58 \mathrm{~cm}$ ). Beide Nasenlöcher befinden sich dicht vor dem Auge. Die Nasengrube hat in ihrer größten Ausdehnung einen Durchmesser von $14 \mathrm{~mm}$. Im Vergleich zu deren Ausdehnung ist das eigentliche Geruchsorgan (Abb. 10) relativ klein. Es liegt als etwa birn- oder feigenförmiges Gebilde von $7 \mathrm{~mm}$ Länge am Boden der Grube direkt unter der die Nasenöffnungen trennenden Haut, ist daher von außen nicht ohne weiteres sichtbar.

Wenn auch das Organ an sich relativ klein ist, so ist in demselben jedoch durch reiche Faltenbildung eine enorme Vergrößerung des olfactorischen Epithels geschaffen worden. Um einen medianen Wulst, in den von hintenoben der Olfactorius eintritt, gruppieren sich rosettenförmig etwa 25-30 Riechfalten, wie es unsere Abbildung deutlich zeigt.

$\mathrm{B}$ e $\mathrm{z}$ a h n ung. Außerordentlich interessant ist die Bezahnung bei $H$. isl., die im wesentlichen der bei $H$. med. entspricht. Zähne finden wir auf dem Praemaxillare (Abb. 21), Dentale (Abb. 11), Palatinum (Abb. 12) und den Pharyngobranchialia. Die etwa $1 \mathrm{~mm}$ langen konischen Zähnchen sind nicht wie bei $H$. med. in Reihen angeordnet, sondern stehen ganz unregelmäßig. Ihre Zahl geht bei Praemaxillare und Dentale in die Tausend. Beim Palatinum ist nur der vordere Rand bezahnt.

Bei geschlossenem Maul stehen sämtliche Zähnchen von Praemaxillare und Dentale außerhalb der Berührungslinie beider Knochen, also a u B e r $\mathrm{h}$ a l b der Mundhöhle. Diese Art der Bezahnung ist zweifellos etwas Außergewöhnliches, pflegen doch normalerweise die Zähne innerhalb der Mundhöhle zu stehen. Ob man diese Besonderheit als Anpassung an eine besondere Lebens- bzw. Ernährungsweise auffassen muß, d. h. ob evtl. mit der so gebildeten Raspel - in Verbindung mit dem rauhen Wulst zwischen den Unterkiefern - festsitgende Nährtiere abgeschabt werden, vermag noch nicht gesagt zu werden, zumal Mageninhaltsuntersuchungen ergaben, daß sich die Tiere von kleinen Fischen - erkannt wurde blauer Wittling (Gadus poutassou) von etwa 15-20.cm Länge -, Mysideen, Crangon und Pandalus ernähren. $\mathrm{Ob}$ 
dieses aber die übliche Nahrung ist, läßt sich natürlich auf Grund einiger weniger Beobachtungen noch nicht entscheiden.

Die Bezahnung der Pharyngobranchialia erstreckt sich auf die Innenflächen der ersten drei Paare (beim 4. Kiemenbogen fehlen die Pharyngobranchialia). Die Zähnchen sind von gleicher Form wie auf den Kiefern, nur etwas länger und nicht so engstehend.

Die äußere Kiemenöffnung ist sehr weit und reicht vom Ansat der Linea lateralis bis zum Isthmus. Wie bei den beiden anderen Arten finden wir bei unseren Fischen 8 Radii brancheostegi und 4 wohl ausgebildete Kiemenbögen (Abb. 14). Auf dem ersten Kiemenbogen finden wir 15 Reusenfortsäze bis zu maximal $3 \mathrm{~cm}$ Länge, auf dem zweiten sind es 14 bis zu etwa $2 \mathrm{~cm}$ Länge, auf dem dritten 13 bis zu $8 \mathrm{~mm}$, während auf dem vierten als Reste nur noch 8 leistenartige Erhebungen von $2 \mathrm{~mm}$ Höhe auf der Innenseite der Bögen zu erkennen sind.

Die letgte Kiemenspalte ist außerordentlich eng, wie es von VAILlanT auch für $H$. med. festgestellt wurde. Die Kiemenblättchen sind doppelt und verhältnismäßig kurz ( $8-13 \mathrm{~mm}$ lang).

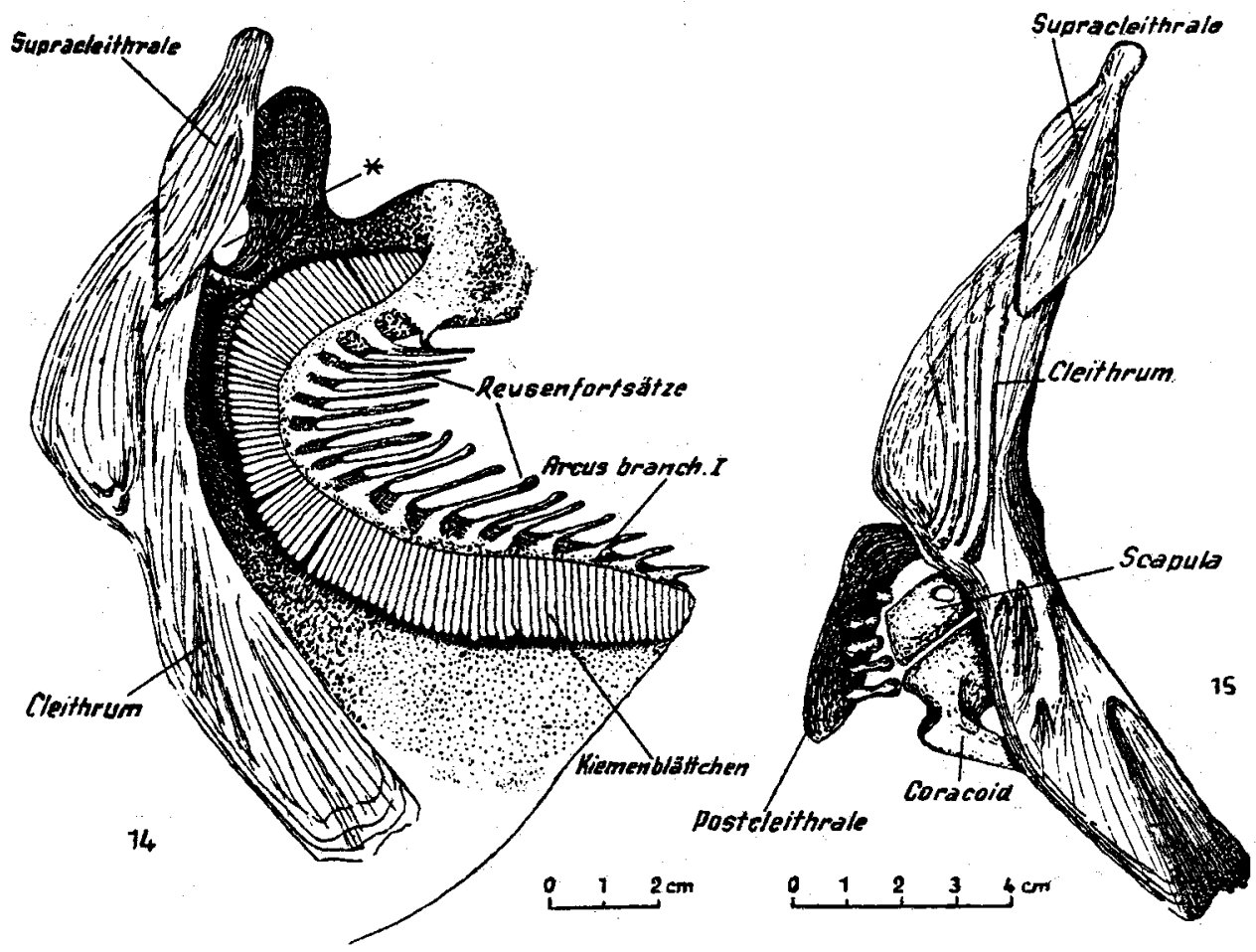

Abb. 14-15. Hoplostethus islandicus, n spec.: 14. Kiemenhöhle rechts (Cleithrum und Surpacleithrale freigelegt) * an dieser Stelle liegt die Schwimmblase der Kiemenhöhlenwand an (s. S. 84); 15. Schultergürtel rechts.

Wie bei den meisten Beryciformes finden wir bei $H$. isl. auch eine a $\mathrm{k}$ zessoris che $\mathrm{K}$ i em e, die aber nicht wie bei $H$. med. unter dem Operculum, sondern unter Praeoperculum und Hyomandibulare sitgt. Sie besteht aus etwa 40 bis zu 1,5 cm langen, aufwärts gerichteten und von einer horizontal verlaufenden Leiste ausgehenden schmalen Blättchen. Sie erstreckt sich vom 
proximalen Rand des Operculums nach vorne in einer Länge von ca. $4 \mathrm{~cm}$, und zwar ungefähr auf der Höhe der Gelenkstelle zwischen Operculum und Hyomandibulare.

Mund und Kiemenhöhle sind von einer schwarzen Schleimhaut ausgekleidet, wie es von den beiden anderen Arten auch bekannt ist.

\section{Die Flossen.}

Neben der für alle Hoplostethusarten charakteristischen Zahl von 6 Hart(Stachel-) strahlen finden wir in der D bei $H$. isl. noch $16-18$ Weich-oder Gliederstrahlen, gegen $12-15$ bei $H$. med. und 17 bei $H$. atl., bei dem allerdings die Hartstrahlen, wie auch an den übrigen Flossen, nur sehr schwach ausgebildet sind. Die Flosse beginnt über der 4 . oder 5. und endet etwa über der 20. Schuppe der Seitenlinie.

Die sechs Hartstrahlen, von denen der erste nur wenige Millimeter groß ist, werden caudalwärts sukzessive länger. Die sich anschließenden Weichstrahlen nehmen bis zum dritten noch geringfügig an Länge gegenüber dem lez̧ten Hartstrahl zu und werden vom vierten an wieder langsam kürzer. Der Hinterrand der $\mathrm{D}$ ist fast halbkreisförmig abgerundet, bildet also nicht wie bei $H$. med, eine mehr oder weniger ausgesprochene Spitze. Dort, wo die D endet, ist die Rückenkontur wulstartig eingeschnürt, wie es auf Abb. 2 deutlich zu erkennen ist.

Dire $\mathrm{C}$ ist bei weitem nicht so tief eingeschnitten wie bei $H$. med. $18-21$ Weichstrahlen werden dorsal von $7-8$ und ventral von $6-7$ rudimentären Stachelstrahlen, die von vorn nach hinten länger werden, eingesäumt. Der leţte bzw. innerste Stachelstrahl erreicht etwa die halbe Länge des ersten bzw. letzten Weichstrahls. Bei H. med. lautet die Formel der C VIII/18/VII, für H. atl. VII/18/VIII.

Außer drei Hartstrahlen sind in der A noch 11-12 Weichstrahlen zu finden, bei $H$. med. sind es $8-11$, bei $H$. atl. aber sind neben 11 Weichstrahlen nur zwei Hartstrahlen vorhanden. Die Flosse beginnt unter dem 8. bis 9. Weichstrahl der D und endet mit letgterer auf gleicher Höhe. Der erste Hartstrahl ist sehr kurz (noch nicht $1 / 2 \mathrm{~cm}$ ), der zweite ist um mehr als das Doppelte länger und der dritte erreicht die halbe Länge des ersten Weichstrahls. Bei H. med. ist die Außenkontur der A convex, bei $H$. isl. dagegen schwach konkav. Beiderseits wird die A von trapezförmigen größeren Schuppen umsäumt.

Für alle drei Hoplostethusarten lautet die Formel für die $V$ übereinstimmend 1/6. Während aber bei H. med. diese Flosse bis zum After reicht, ist sie bei $H$. isl. wesentlich kürzer und endet bereits auf halber Länge zwischen ihrem Ansatz und dem After. Im Ansabpunkt der V unterschieden sich die beiden Arten ganz wesentlich. Bei H. med. ist sie mehr kehlständig, seţt also noch vor der $\mathrm{P}$ an, dagegen steht sie bei $H$. isl. etwa genau unter bzw. etwas hinter dem Ansatz der P.

Bei $H$. isl. finden wir in der P 18-20 Weichstrahlen; bei H. med. aber nur 15 . $\mathrm{Ob}$ bei $H$. atl., wie Colletr angibt, neben 17 Weichstrahlen noch: 1 Hartstrahl vorhanden ist, scheint mir sehr zweifelhaft. Möglicherweise wurde der erste ungegliederte Weichstrahl als Hartstrahl angesehen. Bedeutende Unterschiede bestehen in der Länge der $\mathrm{P}$ bei allen drei Arten. Bei H.med. reicht diese Flosse fast bis zum Ende der A, bei $H$. atl. ist sie kürzer, reicht aber über den After hinaus, während bei den isländischen Fischen der After 
kaum erreicht wird. Auch in der Stellung der $\mathrm{P}$ ergeben sich Unterschiede: bei $H$. med. liegt sie fast horizontal, weist dagegen bei $H$. isl. schräg nach hinten oben.

Die Weichstrahlen der D, A, V und weniger der C und P, weisen Längsleisten auf, die mit nach auswärts gerichteten kleinen, stumpfen Höckern besezt sind, die den ganzen Flossen ein sehr rauhes Gepräge verleihen.

Wenn wir noch einmal die Flossenformeln der drei atlantischen Hoplostethusarten einander gegenüberstellen, dann bekommen wir folgendes Schema:

\begin{tabular}{|c|c|c|c|}
\hline & mediterraneus & H. atlanticus & H. islandicus \\
\hline D & $\mathrm{VI} / 12-15$ & $\mathrm{VI} / 17$ & $\mathrm{VI} / 16-18$ \\
\hline C & VIII/18/VII & VII/18/VIII & VII-VIII/18-21/VI-VII \\
\hline A & III $/ 8-11$ & $11 / 11$ & III/1 1-12 \\
\hline V & $\mathrm{I} / 6$ & $\mathrm{I} / 6$ & $\mathrm{I} / 6$ \\
\hline$P$ & 15 & $\mathrm{I} / 17$ & $18-20$ \\
\hline
\end{tabular}

\section{Das Skelet}

Wenn wir das Skelet von $H$. isl. betrachten, so fällt zuerst auf, daß es außerordentlich leicht, aber trotgdem stabil gebaut ist, vor allem das Exoskelet des Schädels. Das wird dadurch erreicht, daß die Knochenelemente keine kompakte Masse bilden, sondern schwammartig aufgelockert sind und mehr oder weniger große, z. T. durch Fett ausgefüllte Räume zwischen sich frei lassen.

Das Axialskelet bietet gegenüber dem normalen Teleostiertyp nichts wesentlich Neues, nur sei darauf hingewiesen, daß die Rippen außerordentlich dünn sind und nur etwa die Hälfte der Leibeshöhle umfassen. Man zählt bei unseren Fischen 13 Rumpf- und 14 Schwanzwirbel, insgesamt also 27 (ohne Urostyl), während $H$. med. nach VAILLANT deren 24 (10 plus 14), nach LE GALl aber 26 ( 11 plus 15) besiten soll. Ob bei leţterer Angabe das Urostyl eingeschlossen ist, vermerkt LE GaLL nicht.

Wenn das Schädelskelet von $H$. med. auch von Supino ausführlich beschrieben wurde, so lohnt es sich doch, auf das des $H$. isl. einzugehen, da es gegenüber ersterem doch mancherlei Abweichungen aufweist. Vor allem scheint mir dies auch notwendig, da über das Cranium dieser urtümlichen Fische noch allzu wenig bekannt ist.

Surino hat vor allem auf die Beschreibung jedes einzelnen Knochens Wert gelegt und konnte dabei auf eine bildliche Wiedergabe weitgehend verzichten. Ich möchte mich im Gegensaty hierzu im Text nur sehr kurz fassen und die Abbildungen für sich sprechen lassen. Vor allem kommt es mir darauf an, die bestehenden Unterschiede zwischen den Arten herauszustellen.

Das Neurocranium von $H$. isl. weist im Gegensat zu der Mehrzahl der bekannteren Fische und auch im Vergleich zu H.med. eine ungewöhnliche Größe auf. Das hängt damit zusammen, daß die von den einzelnen Knochen dieses Skeletteils eingeschlossene Schädelhöhle nicht nur zur Aufnahme des Gehirns - dieses ist überraschend klein - und des stato-akustischen Apparates dient, sondern zusätglich noch eine andere Aufgabe übernommen hat, die wohl in dieser Form einzigartig unter den Fischen dasteht. Nur ein sehr kleiner Teil der Schädelhöhle dient seiner ursprünglichen Bestimmung. Der weitaus größte Raum aber wird von einer Fettreserve ausgefüllt, die hier in Form einer weißen, speckartigen Gewebsmasse deponiert ist. Aus dem Schädel 
eines Tieres von $59 \mathrm{~cm}$ konnte beispielsweise $28 \mathrm{~g}$ reines Fett gewonnen werden! Weiter unten wird im Zusammenhang mit den übrigen Fettreserven noch einmal näher darauf einzugehen sein.

Man kann die Schädelhöhle in zwei Abschnitte unterteilen, nämlich in einen proximalen, seitlich stark komprimierten interorbitalen Teil (Abb. 23,v), der caudal, durch je eine Crista der Alisphenoide eingeengt, in den wesentlich größeren und geräumigen distalen Abschnitt (h) übergeht, der in der Hauptsache von den Knochen der Regio occipitalis und der Oticalregion umschlossen wird.

Die Vergrößerung. der Schädelhöhle bringt es mit sich, daß alle an: ihrer Bildung beteiligten Knochen relativ groß sind und z. T. auch eine Lageverschiebung erfahren haben.

Basi- (Abb. 26, B. occ.) und Exoccipitalia bieten gegenüber H. med. nichts Besonderes, dagegen erscheint das Supraoccipitale (Abb. 26, S. occ.) in seiner Dorsalpartie stark vergrößert. Es bildet mit einer nahezu kreisrunden. Scheibe, der eine hohe Crista aufsitgt, das hintere Dach der Schädelhöhle. Ebenso wie bei $H$. med. ist dieser Knochen nicht an der Bildung des Foramen magnum beteiligt; legteres wird ausschließlich von den Exoccipitalia umschlossen.

Dem vorderen Rande des Exoccipitale sitgt fest verkeilt das Opistoticum auf, das SupINo für H. med. nicht erwähnt. Es ist ein kleiner, dreiseitiger Knochen, der vorne unten an das Prooticum, vorne oben an das Pteroticum grenzt. An der Grenze zwischen Opistoticum und Pteroticum ist, von beiden Knochen gemeinsam gebildet, eine schwache Gelenkpfanne für den stielaritigen Teil des Posttemporale (Abb. 17; Abb. 26, Pt.) zu erkennen.

Die breite Gelenkpfanne für das Hyomandibulare (Abb. 26, Hyom.; Abb. 16) wird in ihrem weitaus größten caudalen Abschnitt vom Pteroticum, in ihrem kleineren proximalen Abschnitt gemeinsam von Pro- und Sphenoticum gebildet.

Das im Querschnitt V-förmig gebogene Pteroticum trägt auf der lateralen, vorspringenden Crista zwei dünne, ringförmig gegeneinander gebogene Fortsätze, durch die der caudale Schleimkanal in das Praeoperculum eindringt.

An der vorderen Begrenzung des distalen, weiten Teiles der Schädelhöhle, sind basal- und lateralwärts die Prootici, medial das Y-förmige Basisphenoid und dorsolateral die Alisphenoide beteiligt. Letztere haben gleichzeitig teil an der distalen Begrenzung des interorbital gelegenen Teiles der Schädelhöhle. Im Wesentlichen wird dieser Abschnitt lateralwärts von den Orbitosphenoiden, die hier wie bei H. med. an der Basis zu einem einheitlichen, im Querschnitt V-förmigen Knochen verwachsen sind, begrenzt. Den proximalen Abschluß der Schädelhöhle stellt das Mesethmoid (Abb. 19) her, das caudalwärts tief ausgehöhlt ist. Es stoßen also Orbitosphenoid und Mesethmoid direkt aneinander. Gegenüber H. med. ist also bei unseren Fischen das Orbitosphenoid, das dort noch Verbindung mit dem Prooticum hat, sehr weit nach vorne verlagert.

Im Anschluß an das Supraoccipitale bildet nach vorne zu jederseits das Frontale (Abb. 26, Fr.) - bei H. med. ist nur e in Frontale vorhanden - und daran oralwärts anschließend das Praefrontale (Abb. 26, Prfr.) den dorsalen Abschluß der Schädelhöhle. Das hinten seitlich dem Frontale ansitzende Parietale (Ab. 26, Par.) schließt im Schädeldach noch eine kleine Lücke zwischen Supraoccipitale, Epi-, Pter- und Sphenoticum. Am seitlichen unteren Rande des Frontale, der dem Sphenoticum aufliegt, finden wir noch einen kleinen 
Deckknochen, der sich von hier bis zum Pteroticum hinzieht und dieses sowie das Sphenoticum teilweise bedeckt. Seiner Lage nach müssen wir ihn als Petrosum (Abb. 26, Petr.) ansprechen. Zwei hakenartig gegeneinander gerichtete Lamellen dieses Knochens bilden den Durchlaß für den suborbitalen Schleimkanal. Dieser Knochen fehlt bei H. med. An das Parietale schließt sich caudalwärts je ein Postfrontale (Abb. 17) an, das die Verbindung mit dem dorsalen, schirmartig verbreiterten Teil des Posttemporale (Abb. 18) herstellt. Auch das Posttemporale ist bei SuPiNo für H. med. nicht erwähnt.

Während das seitlich stark ausladende Frontale, in Verbindung mit einer Crista des Sphenoticums, das Dach der Orbita bildet, wird diese hinten, unten und vorne, bis auf eine kleine Lücke hinter den Nasenöffnungen, vom Infraorbitalring (Abb. 20 u. 26, I. orb.) begrenzt. Dieser Ring besteht aus fünf Einzelknochen, von denen der dritte am größten, der leţte am kleinsten ist. Während auf den Infraorbitalia I, IV u. V sich jeweils e in e Knochenbrücke radialwärts erstreckt, finden wir auf dem Infraorbitale II zwei unterbrochene und auf I. orb. III eine hintere durchgehende und eine vordere unterbrochene Brücke. Alle diese nach außen vortretenden Leisten sind relativ viel breiter als bei H. med. Mit ihren distalen Rändern grenzen die drei hinteren Ringelemente an das Praeoperculum.

Daß die Oberflächen all dieser Deckknochen reich skulpturisiert sind, wurde bereits oben vermerkt.

Im Zusammenhang mit der Besprechung des Neurocraniums sei noch erwähnt, daß die Statolithen ("Sagitta" aus dem Sacculus) von H. isl. sehr groß (etwa $2 \mathrm{~cm}$ lang und $1 \mathrm{~cm}$ breit) und von bizarrer Gestalt sind, wie es in Abb. 13 deutlich zum Ausdruck kommt.

Visceralskelet. Während die Gaumenknochen (Palatinum, Ectopterygoid, Entopterygoid und Metapterygoid, in Abb. 26 teilweise durch den Infraorbitalring verdeckt) sowie das Quadratum und der Kieferstiel (Hyomandibulare und Symplecticum, Abb. 16) in Form und Lage nicht von H. med. abweichen, ist der Oberkiefer (Abb. 21) etwas anders gestaltet. Sowohl beim Praemaxillare als auch bei dem etwas längeren Maxillare ist die vordere Außenkante gleichmäßig konvex gebogen, dagegen bei $H$. med. in sich noch flammenlinienartig geschwungen. Das dem Maxillare aufliegende Supramaxillare ist bei $H$. isl. robuster und relativ größer als bei $H$. med.

Im Unterkiefer (Dentale, Articulare und Angulare, Abb. 11 u. 26) sind keine wesentlichen Unterschiede zu $H$. med. zu finden. Auch im Bau des Hyobranchialskelets finden wir in beiden Fällen in etwa die gleichen Verhältnisse. Es sei hier nur noch einmal auf die außergewöhnlich massige Entwicklung des Hyalbogens (Abb. 16), vor allem des Ceratohyale, hingewiesen.

Die Elemente des Kiemendeckels: (Abb. 16 u. 26) weisen wieder größere Abweichungen auf. Das Praeoperculum (P.op.) hat bei H. med. zwischen seinen vertikalen seitlichen, vorspringenden Leisten $\mathrm{v}$ i e $\mathrm{r}$ vollständige Knochenbrücken, dagegen finden wir bei $H$. isl. nur $\mathrm{z}$ w e i vollständige, und eine weitere obere ist andeutungsweise vorhanden. Vor allem aber fehlt $H$. isl. der am Basalteil dieses Knochens vorhandene, caudalwärts gerichtete starke Dorn, der sowohl für $H$. med. wie auch für $H$. atl. so charakteristisch ist.

Das Operculum (Op.) ist ein großer flacher Knochen von vogelflügelartiger Gestalt, ja man könnte die auf seinem unteren Teil vorhandenen 

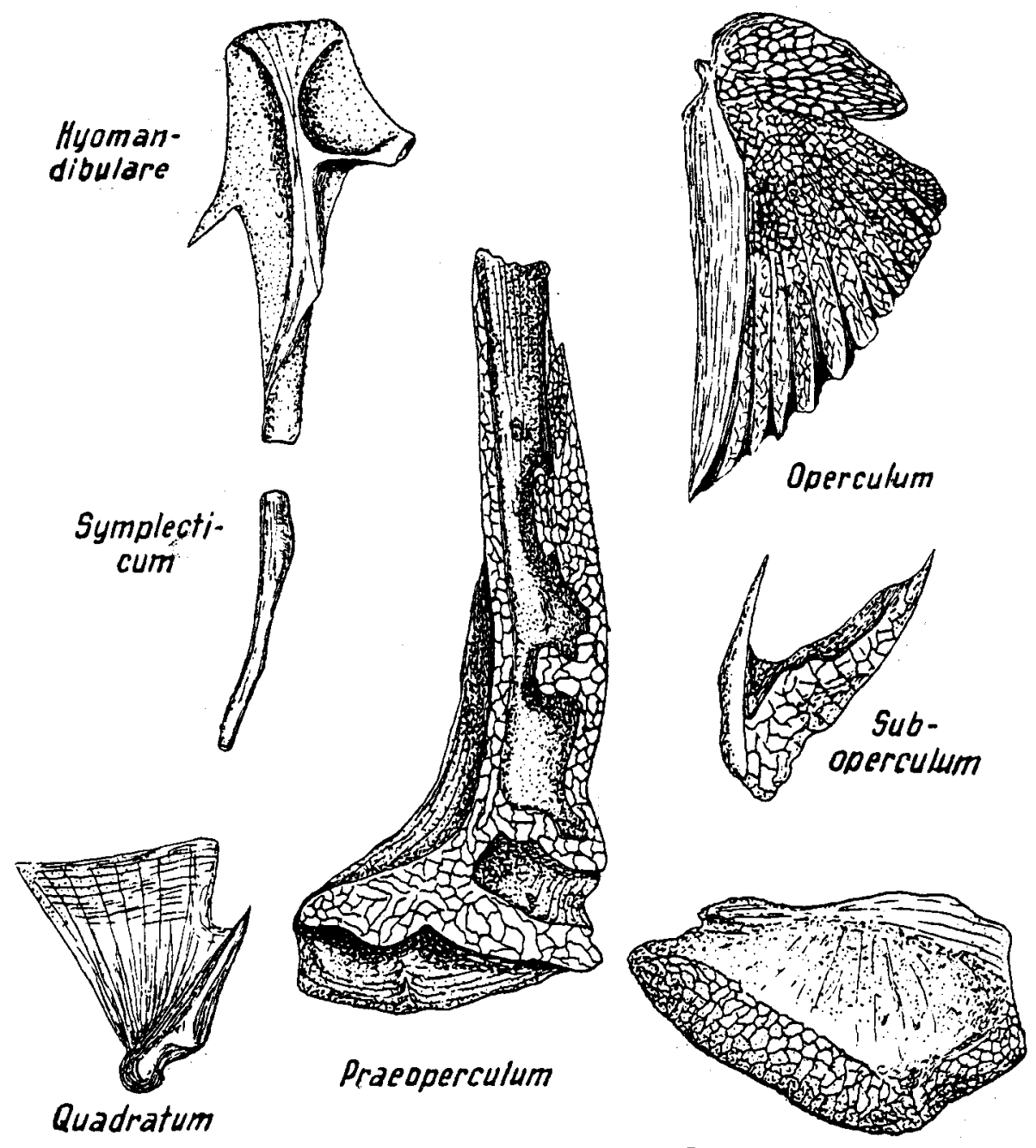

Interoperculum

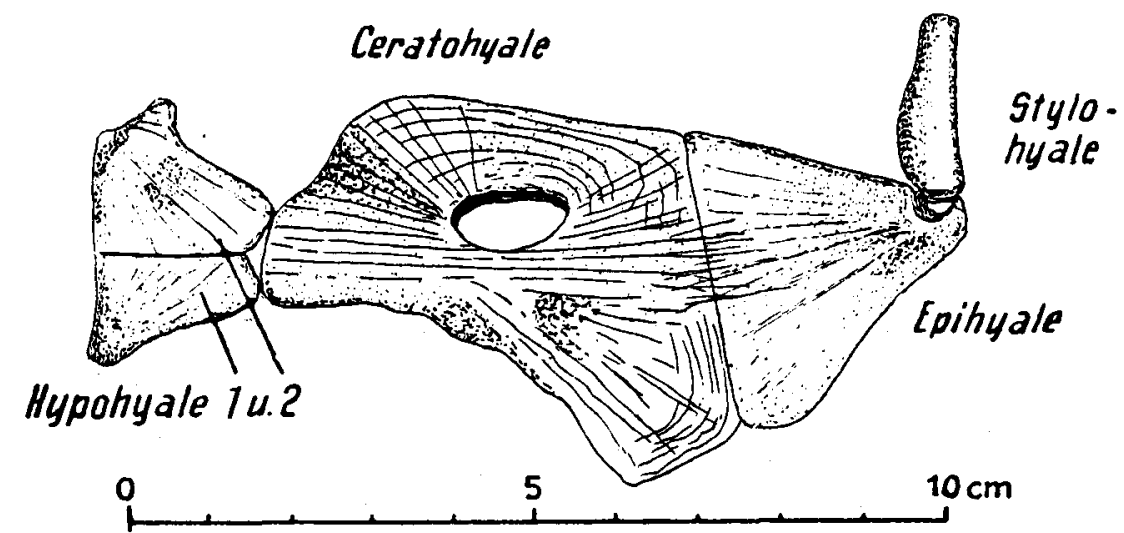

Abb. 16. Hoplostethus islandicus, n. spec., Kieferstiel, Kiemendeckelknochen, Hyalbogen. 
radialen Leisten direkt mit den Federn des Flügels vergleichen. Es liegt in seiner ganzen Ausdehnung frei zutage.

Das Suboperculum (S. op.) ist sehr klein. Zum größten Teil ist es vom Operculum und Praeoperculum verdeckt, nur eine schmale caudale, dem Hinterrand des Operculums anliegende Leiste liegt frei.

Das Interoperculum (I. op.) ist ein sehr großer Knochen, der aber nur mit seinem schmalen ventralen Rand frei liegt, im übrigen aber vom Basalteil des Praeoperculums verdeckt ist.

Im Anschluß an den Schädel sei noch kurz auf den Schultergürtel (Abb. 15, 17, 18, 26) eingegangen, den Supino nicht beschrieben hat. Die

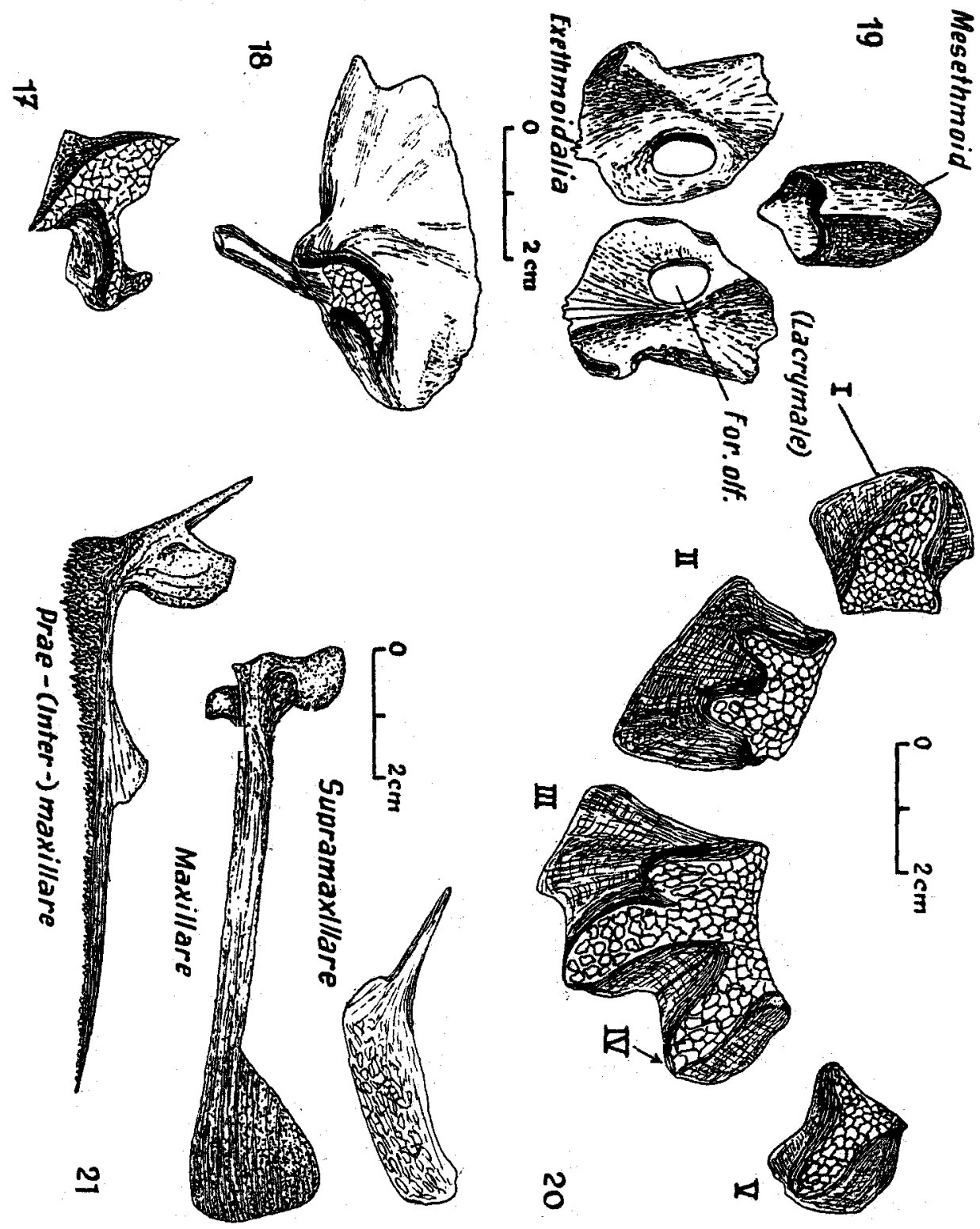

Abb. 17-21. Hoplostethus islandicus, n. spec., Teile des Kopfskelets: 17. Postfrontale; 18. Posttemporale; 19. Ethmoidalia; 20. Infraorbitalia links; 21. Oberkiefer links. 
Verbindung des Schultergürtels mit dem Neurocranium wird, wie bereits kurz angedeutet, durch das Posttemporale ( $\mathrm{Pt}$.) hergestellt. Dieses besteht aus einer breiten ovalen Platte und einem, am unteren hinteren Rande ansetzenden, schräg nach vorn innen zeigenden Stiel, der mit Pter- und Opistoticum gelenkig verbunden ist. Zwischen Parietale und Posttemporale wird die Verbindung durch das dazwischenliegende Postfrontale hergestellt. Für die Artcharakteristik ist besonders wichtig zu bemerken, daß H.isl. ke in e n Posttemporal-(Suprascapular-)Dorn besitgt, wie die beiden anderen Arten, vielmehr ist der Hinterrand dieses Knochens gleichmäßig abgerundet. Auch das Posttemporale trägt durch zwei vorspringende Leisten zur Skulpturierung des Schädels bei.

Das Supracleithrale (Abb. 26, Scl.) lenkt hinter dem Stiel am Posttemporale ein und legt sich mit seinem verbreiterten und ventral sich wieder verjüngenden Abschnitt dem oberen Teil des Cleithrum (Clavicula, Cl.) von außen auf. Das langgestreckte Cleithrum bildet mit seinem proximalen Rand die hintere Begrenzung der Kiemenhöhle (Abb. 14). Seiner schmalen Mittelpartie liegen von innen Scapula (Scp.) und Coracoid (Co.) auf. Das Postcleithrale (Pcl.) liegt mit seiner stielartigen Verlängerung dem oberen breiten Abschnitt des Cleithrum auf, während sein verbreiterter Haupteil frei unter den Radien der Pectoralflosse liegt. Von den fünf vorhandenen Radien steht der untere und längste mit dem Coracoid, die übrigen vier mit der Scapula in Verbindung.

\section{Innere Organis ation}

Zur Abrundung des Bildes sei auch auf die innere Organisation kurz hingewiesen, allerdings sollen und können diese Organe hier nicht vergleichendanatomisch behandelt werden; das soll in einer späteren Arbeit nachgeholt werden. Ich werde mich im wesentlichen darauf beschränken, die Befunde mehr oder weniger kommentarlos wiederzugeben und nur dort, wo möglich auf die Unterschiede zu H. med. hinweisen.

Die Leibeshöhle ist wie bei allen Trachichthyidae-Arten mit einem schwarzen Peritoneum ausgekleidet.

Das $\mathrm{H}$ e $\mathrm{r} z$ von $H$. isl. (Abb. 25) liegt in einem umfangreichen Fettgewebe eingebettet und ist relativ groß. Das dünnhäutige Atrium mündet an der linken Seite in den Ventrikel, dessen Lumen nicht glattwandig, wie sonst bei Teleostiern üblich, sondern in ähnlicher Weise wie bei den Selachiern stark gelappt ist. Am Úbergang vom Ventrikel zum Conus arteriosus finden wir drei taschenartige Herzklappen. Das Lumen des Conus arteriosus wird durch radiale Lamellen stark eingeengt, ist also ebenfalls nicht glattwandig wie es bei den Teleostiern normalerweise der Fall ist.

Der Verdauungstraktus beginnt mit einem sehr muskulösen und faltenreichen Oesophagus, der in einen seitlich stark komprimierten großen Magen übergeht. Auch dieser weist reiche Faltenbildung auf, namentlich in seinem vorderen unteren Teil, wo sich der Pylorus anschließt. Der Darm bildet zwei große Schleifen. Am Pylorus mündet zunächst der Gallengang; die Gallenblase selbst liegt im Hinterrand des linken Leberlappens eingebettet. Beachtenswert ist die Größe der Leber; denn gewöhnlich ist diese bei einem „Fettfisch“ (s. weiter unten) sehr klein.

Wie bereits Cuvier \& Valenciennes für $H$. med. festgestellt hatten, ist die Zahl der Appendices pyloricae sehr groß. Eine Zahl wurde aller- 
dings nicht angegeben. Nach VAILlANT dagegen sind es nur etwa 15 („une quinzaine") Schläuche. Im Gegensat hierzu ist die Zahl der App. pyl. bei H.isl. außerordentlich groß. Sie liegen hier nicht frei voneinander, sondern sind unter einer gemeinsamen bindegewebigen Haut verborgen und auch untereinander durch Bindegewebe verbunden, so daß der Eindruck eines einheitlichen Organs entsteht. Jeweils 2-5 dieser Schläuche sind zu einem Büschel mit gemeinsamer Mündung zusammengefaßt. Bei einem Tier zählte ich 13.5 solcher Büschel mit insgesamt etwa 350 Einzelschläuchen. Diese Büschel sitzen auf der ersten Darmschlinge in einer Ausdehnung von ca. $15 \mathrm{~cm}$ dem Darm an. Pankreas und Langerhanssche Inseln sind wohl ausgebildet und liegen hintereinander dem Mitteldarm auf.

Der Erhaltungszustand der $\mathrm{N}$ i e r e $\mathrm{n}$ war recht schlecht, so daß eine gerauere histologische Untersuchung an ihnen nicht mehr möglich ist, was an sich insofern bedauerlich ist, als wir bei unseren Tieren diese Organe in einer ganz anderen - wahrscheinlich primitiveren - Form antreffen als bei anderen Fischen.

Der caudale Abschnitt der Nieren ist zu einem großen unpaaren Organkomplex verschmolzen, der über den caudalen. Teilen der Ovarien bzw. Hoden liegt. Nach vorne erstrecken sich die Nieren jeweils in einem Kanal seitlich unter dem Basioccipitale bis in die Oticalregion. Ob dieser proximale, außerhalb der Leibeshöhle liegende Teil der Nieren als Rest des Pronephros (.,Kopfniere“) aufzufassen ist, vermag nur durch histologische Untersuchung geklärt zu werden.

Die Ovarien sind gegenüber anderen Fischen nicht abweichend gebaut; der Oviduct mündet zusammen mit dem Ureter auf einer kleinen lap-
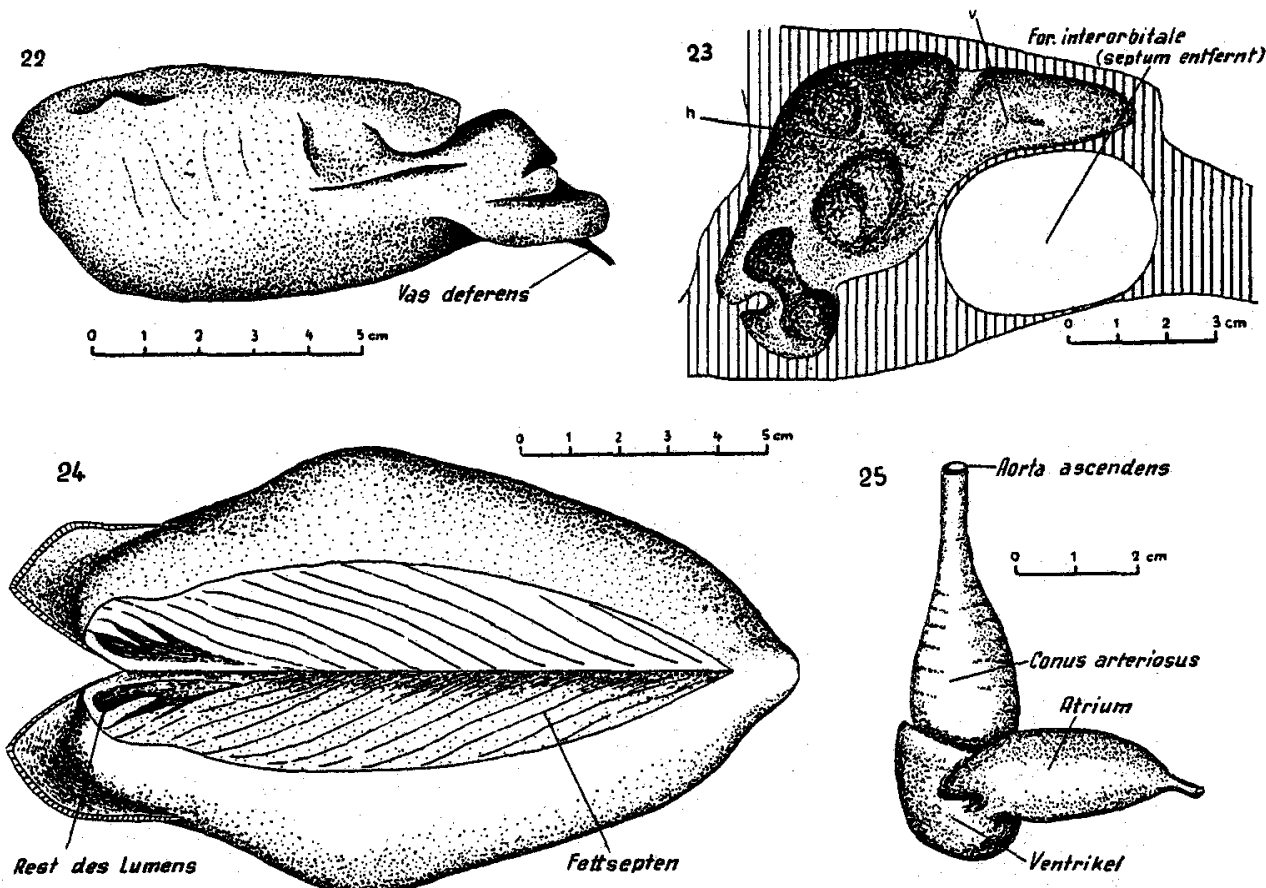

Abb. 22-25: Hoplostethus islandicus, n. spec., Schädel im Längsschnitt und innere Organe: 22. Hoden links, Außenansicht; 23. Längsschnitt durch den Schädel, hinten nicht genau median getroffen; 24. Schwimmblase, ventral geöffnet; 25. Herz: 


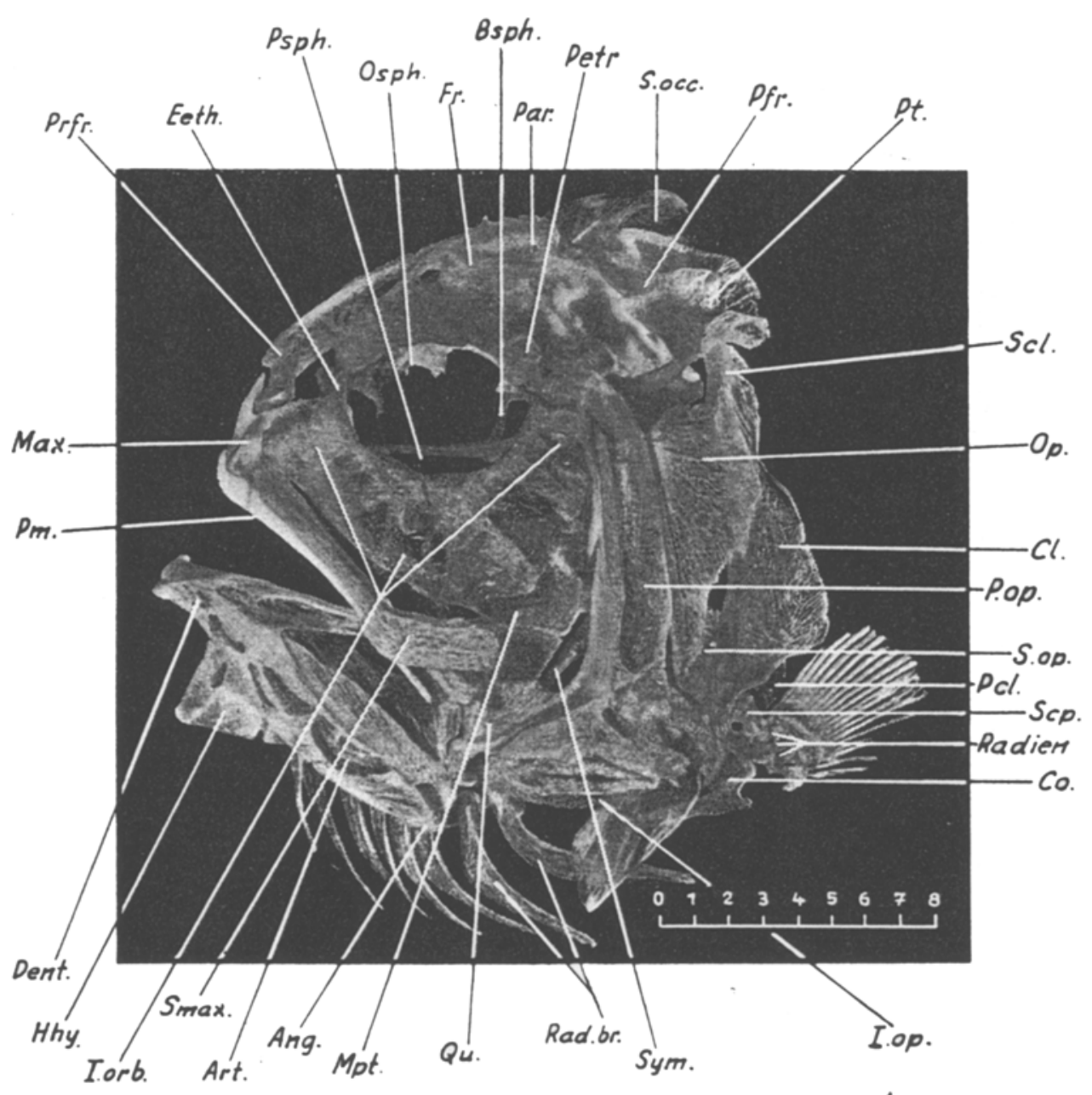

Abb. 26. Hoplostethus islandicus, n. spec., Schädelskelet.

(Foto Biolog. Anstalt Helgoland - BEISSERT)

pigen Genitalpapille. Die Zahl der etwa $1,1 \mathrm{~mm}$.großen ziegelroten Eier beträgt 200000 bis 250000 . Über die Struktur der Eier selbst ist nichts mehr auszusagen, da der Erhaltungszustand zu schlecht war.

Ganz außergewöhnlich groß sind die $\mathrm{H}$ od e $\mathrm{n}$ (Abb. 22). Sie erreichen auf dem Reifestadium V nathezu die Größe einer Männerhand. Beide zusammen nehmen in reifem Zustande mehr als ${ }^{2 / 3}$ der Leibeshöhle ein und wogen bei einem Männchen von $58 \mathrm{~cm}$ Totallänge (Gewicht $3,69 \mathrm{~kg}$ ) $253 \mathrm{~g}$, d. h. ca. 6,6\% des Gesamtgewichtes. Die.Vasa deferentia münden zusammen mit dem Ureter auf einer, ähnlich wie im weiblichen Geschlecht gestalteten Genitalpapille aus, so daß die Geschlechter nicht an den Papillen zu unterscheiden sind, wie es etwa bei Sebastes marinus ohne weiteres möglich ist.

Die sehr große Schwimmblase (Abb. 24) hat ihre eigentliche Aufgabe, ein hydrostatisches Organ zu sein, so gut wie gänzlich eingebüßt; denn ihr Lumen ist durch enorme Fettmassen auf den proximalen dorsalen Abschnitt des Organs zusammengedrängt, bei einzelnen Tieren sogar gänzlich verloren gegangen. Eine hydrostatische Funktion kann ein solches Organ nicht mehr 
ausüben. Hierin unterscheidet sich $H$. isl. ganz grundlegend von den beiden anderen Arten, denen eine wohl ausgebildete Schwimmblase mit großem „roten Körper" zukommt, ja, vielleicht sogar von allen anderen Fischen; denn so sehr man auch die Literatur studieren mag, nirgends findet man auch nur im entferntesten derartige Verhältnisse verzeichnet. Es muß noch besonders hervorgehoben werden, daß diese Schwimmblase in ihrem proximalen Abschnitt in engster Beziehung zur Kiemenhöhle steht, von der sie nur durch die Schleimhaut der Kiemenhöhle getrennt ist. Es gelingt daher kaum, die Schwimmblase aus der Leibeshöhle zu trennen, ohne entweder sie oder aber die Haut der Kiemenhöhle zu beschädigen. (Auf eine solche Beschädigung der Kiemenhöhle ist auch das in unserer Abb. 14 eingezeichnete Loch im caudalen oberen Abschnitt der Kiemenhöhle zurückzuführen.) Welche Bedeutung dieser engen Verbindung zukommt, muß einstweilen ungeklärt bleiben.

\section{Fettgehalt}

Wie wir sahen, finden wir bei $H$.isl. an zwei ganz ungewöhnlichen Stellen Fettreserven angehäuft, nämlich in der Schädelhöhle und in der Schwimmblase. Bei dem Fett im Schädel handelt es sich um eine sehr feste, speckartige weiße Masse, die nach den Bestimmungen von Herrn Dr. Richter, Institut für Meeresforschung-Bremerhaven, zu 12,5\% aus Wasser und 87,3\% aus Fett besteht (Säurezahl 0,3, Refraktionszahl bei $20^{\circ} \mathrm{C}$ 1,464, Jodzahl 82,4. Verseifungszahl 154). Das Fettgewebe der Schwimmblase ist von etwas weicherer Konsistenz, weist aber einen etwas geringeren Wassergehalt (10,2 \%) auf. Die Kennzahlen sind kaum von denen des Kopffettes unterschieden; jedoch sind darin $45,3 \%$ unverseifbare Anteile enthalten, die bei $20^{\circ} \mathrm{C}$ flüssig sind. Durch Erhitzen läßt sich sowohl aus dem Fettgewebe des Kopfes wie auch aus dem der Schwimmblase ein vollkommen kristallklares, geruch- und geschmackfreies Oll gewinnen.

Dieses sind aber nicht die einzigen Fettreserven, über die $H$. isl. verfügt; denn auch die Muskulatur und die Leber sind als sehr fettreich zu bezeichnen. Nach den Bestimmungen von Herrn Dr. Richter, der mir freundlicherweise diese Daten zur Verfügung stellte und dem auch hier dafür gedankt sei, enthält das Filet neben $74,0 \%$ Wasser und 18,2\% Proteinen noch $7,8 \%$ Fett, in einem zweiten Falle neben dem gleichen Wassergehalt 13,1\% Proteine und $10,8 \%$ Fett. Dieser Fettgehalt entspricht nach WILLE und LUECKE in etwa dem mittleren Fettgehalt des Heringsfleisches. In der Leber stellte Dr. Richter neben $68,8 \%$ Wasser einen Fettgehalt von $16,9 \%$ fest (Refraktionszahl bei 20. C 1,485 ).

Auch die Knochen enthalten große Fettmengen, die leider aus technischen Gründen noch nicht wertmäßig erfaßt werden konnten. Ich kann aber darauf hinweisen, daß sie, nachdem sie 4 Tage in Benzol gelegen hatten - bei sonstigen Fischen genügen $1-2$ Tage - noch nicht fettfrei waren, sondern schon nach wenigen Wochen gelb wurden, so daß eine nochmalige Behandlung mit einem Benzin/Athergemisch von 4 Tagen Dauer erforderlich war, um sie vollkommen fettfrei zu bekommen.

Die Tiere strogen also förmlich von Fett, und es ist tatsächlich so, daß man fettige Finger bekommt, wo immer man das Tier anfassen mag. Wenn wir auch keine Zahlen für den absoluten Fettgehalt des ganzen Fisches geben können, so darf man $H$. isl. doch wohl zu den fettreichsten Fischen zählen. 


\section{Zusammenfassung}

A $11 \mathrm{geme}$ e ne Diagnose.

Körper lang-oval, tropfenförmig, seitlich stark kompreß. Augen mäßig groß. Maul weit und schräg gestellt. Bezahnung auf Kiefer und Palatinum schwach, etwas stärker auf den Pharyngobranchialia. Exoskelet des Schädels dermal gelegen. Entweder mit der ganzen Oberfläche oder mit leistenartigen Brücken treten diese Knochen frei zutage. Dazwischen liegen große schleimgefüllte, durch eine dünne, transparente und poröse Haut abgeschlossene Räume, die untereinander in Verbindung stehen. Oberfläche der Knochen sehr rauh. Infraorbitalring sehr stark ausgeprägt, steht hinten mit dem Praeoperculum in Verbindung. Praeoperculum und Posttemporale (Suprascapulare) ohne Dorn. Scharfer Kiel. Caudalflosse gegabelt. Eine Dorsalflosse.

$$
\text { Spezielle Diagnose. }
$$

Größte Körperhöhe 34,3\% der Totallänge. Schuppen der verschiedenen Körperregionen sehr unterschiedlich in Größe und Form. Freies Ende der Körperschuppen mit schwachen, hinten in einen Dorn endenden Leisten besetgt (ctenoid). Kielschuppen V-förmig mit starkem, rückwärts gerichtetem Dorn. Schuppen der Linea lateralis groß, flügelartig verbreitert, freies Ende bedornt, Basalteil für den Seitenlinienkanal durchbohrt.

Farbe: leuchtend dunkelrot (wie Sebastes marinus). Mundschleimhaut und Peritoneum schwarz.

Kopf: Kopflänge $28,6 \%$, Kopfhöhe $30,3 \%$ der Totallänge. Augendurchmesser 26,7\% der Kopflänge. Hinterrand des Maxillare reicht über die Vertikale des hinteren Augenrandes nach hinten hinaus. Samtartige Bezahnung auf den Außenflächen von Praemaxillare, Dentale und am Vorderrand des Palatinums. Praeoperculum mit zwei Knochenbrücken zwischen den seitlichen Randleisten. Suboperculum sehr klein. Wangen zwischen Maxillare, Infraorbitale III und Praeoperculum beschuppt. Kleine napfförmige Schuppen zwischen den Unterkiefern. Schädelhöhle sehr geräumig, zum größten Teil von einem festen, weißen Fettgewebe ausgefüllt. Gehirn auffallend klein. Statolithen sehr groß und von unregelmäßiger Gestalt. Kiemenöffnung sehr weit. Reusenfortsätze auf den Kiemenbögen, namentlich auf den ersten beiden, sehr lang. Accessorische Kieme auf dem Kiemendeckel unter Praeoperculum und Hyomandibulare.

Schwimmblase sehr groß; ihr Lumen ist durch enorme Fettmassen auf den vorderen Abschnitt der Blase zusammengedrängt, oder auch gänzlich verschwunden. Vordere seitliche Teile der Schwimmblase nur durch die Schleimhaut der Kiemenhöhle von dieser getrennt. Nieren sehr lang, ziehen sich in zwei Kanälen bis in die Oticalregion des Schädels. Magen groß, seitlich komprimiert. Zahlreiche, büschelförmig angeordnete und unter gemeinsamer Haut. liegende Appendices pyloricae (ca. 135 Büschel mit ca. 350 Einzelschläuchen). Ovarien normal. Eizahl 200000 bis 250000 . Eidurchmesser 1,1 mm, Farbe ziegelrot. Hoden außerordentlich stark entwickelt, etwa handgroß im reifen Zustande.

Dorsalflosse: VI/16-18. Stachelstrahlen successive größer werdend. Weichstrahliger Teil nur wenig höher als der stachelige. Vorderer Ansats über der 4.-5. Seitenlinienschuppe. Endet auf gleicher Höhe mit der Analflosse. Am Ende der D Rücken wulstartig erhöht. 
Caudalflosse VII-VIII/19-21/VI-VII. Die rudimentären Stachelstrahlen wesentlich kürzer als die Weichstrahlen.

Analflosse III/11-12. Set5t unter dem 8.-9. Weichstrahl der D an. Die drei Stachelstrahlen sehr kurz und kräftig.

Ventralflosse I/6. Setzt unter oder wenig hinter der Pectoralflosse an, endet ungefähr in der Mitte der Kiellinie.

Pectoralflosse 18-20, symmetrisch, etwas schräg nach oben-hinten stehend, erreicht kaum die Verticale durch den After.

Linea lateralis: $31-32$ Schuppen.

Kielschuppen: 17-18.

Wirbelzahl: 13 Rumpf-plus 14 Schwanzwirbel (ohne Urostyl).

Radii brancheostegi: 8 .

Fundorte: November 1949, Island-Süd (Oeraefagrund), $35 \mathrm{Sm}$ südl. von Ingolfshöfdi, $180 \mathrm{~m}$ Wassertiefe, 5 Exemplare (54-68 cm Länge).

15. I. 1951, Island-Süd, Westteil der Medallandbucht („Schonersbank“), 220-240 m Wassertiefe, 23 Exemplare $(54-63 \mathrm{~cm})$.

19. I. 19.51, Island-Süd, Skafta-Tief (Westl. Oeraefagrund), $220-240 \mathrm{~m}$ Wassertiefe, 8 Exemplare $(56-68 \mathrm{~cm})$.

Wahrscheinlich halten sich die Tiere am Steilhang des isl. Schelfes in kleinen Schwärmen zusammen; denn sie wurden jeweils auf engem Raum gefangen. Ihre Nahrung besteht, soweit man aus dem Mageninhalt schließen darf, aus kleinen Fischen (Gadus poutassou bis ca. $20 \mathrm{~cm}$ Länge), Mysideen, Crangon und Pandalus.

\section{Literatur}

(Die mit *) versehenen Schriften konnten nicht im Original eingesehen werden, sondern sind nur auf Grund von Zitaten anderer Autoren erwähnt, aber der Vollständigkeit halber hier mit aufgeführt.)

*) A g a s s i z, 1899 - Hoplostethus pacificus, n. spec. In: Mem. Mus. Comp. Zool. Harvard College, 24.

*) Alcock, A., 1894 - Natural history notes from H. M. Indian Marine Survey Steamer "Investigator". In: Jour. Asiatic Soc. Bengal, 63, 2 (S. 116).

*) Alcock, A., 1899 - A discriptive catalogue of the Indian deep-sea fishes in the Indian Museum Calcutta (S. 34, Taf. 14, Abb. 3).

A nonym, 1919 - Rés. Camp. Sci. du Prince de Monaco, 52 (S. 46-47).

*) Be eker, 1859 - Tentamen.

*) Bigelow, Farfante, Schroeder, 1948 - Fishes of the Western North Atlantic, $\mathrm{Pt}$. I. In: Sears foundation for marine researche, 1, New Haven. Bolk, Göppert, Kallisch, Lukosch, 1939 - Handb. d. vergl. Anat. d. Wirbeltiere.

*) Bonaparte, 1841 - Catalogine (S. 499).

Brauer, A., 1906 - Wiss. Erg. d. deutschen Tiefs.-Exp. auf d. Dampfer "Valdivia“ 1898/99. Die Tiefseefische, I. Systematischer Teil (S. 285-286).

Canestrini, G., 1870 - Fauna d'Italia, 3. T1., Pesci (S. 73).

*) Cligny, A., 1905 - Poissons des côtes d'Espagne et de Portugal. In: Ann. Stat. Aquicole, Boulogne, N.S. 1 (S. 73)

Collett, R., 1889 - Poissons nouveaux provenant des campagnes de l'Hirondelle, Hoplostethus atlanticus. In: Bull. Soc. Zool. de France, 14, 7 (S. 306).

Cuvier \& Valenciennes, 1829 - Histoire naturelle des poissons, 3 (S. 229), 4 (S. 46.9).

De a n, B., 1895 - Fishes, living and fossil. In: Columbia Univ. Biol. Ser., 3.

Ehrenbaum, E., 1936 - Naturgeschichte und wirtschaftliche Bedeutung der Seefische Nordeuropas. In: Handb. Seef. Nordeuropas, 2.

Franz, V., 1910 - Die japanischen Knochenfische der Sammlung Haberer und Doflein. In: Beitr. Naturg. Ostasiens (S. 31-32). 
Fridriksson, A., $1949^{\circ}$ - Boreo-tended changes in the marine vertebrate fauna of Iceland during the last 25 years. Rapp. Proc. Verb. 125.

Le Gall, J., 1932 - Hoplostethus mediterraneus. In: L. Joubin: Faune Ichthyol. Atl. Nord.

*) German, 1899 - Reports on an exploration off the West coast of Mexico, central and South America, and off the Galapagos Islands. In: Mem. Mus. Com?. Zool. Harvard College, 24.

Goode \& Bean, 1896 - Oceanic Ichthyologie. Report on the results of dredging ect., 36. In: Mem. Mus. Comp. Zool. Harvard College, 5, 22.

Günther, A., 1859 - Catalogue of the acanthopterygian fishes in the collection of the British Museum, 1 (S. 9).

Günther, A., 1886 - Handbuch der Ichthyologie (S. 296).

*) Guichenot, 1850 - Expl. Sci. Algérie, Zool. 5.

Hilgendorf, 1879 - Einige Beiträge zur Ichthyologie Japans. In: Sitz. Ber. Ges. Naturf. Freunde Berlin, 1879, 5.

Holt \& Calderwood, 1895 - Survey of fishing grounds, West coast of Ireland 1890-1891, Report on rarer fishes. In: Sci. Transact. Roy. Dublin Soc., 5, S. 2 (S. 412). Ihle, van Kampen, Nierstrasz, Versluys, 1927 - Vergleichende Anatomie der Wirbeltiere.

Johns on, J.Y., 1866 - Description of Trachichthys Darwinii, a new species of Berycoid fish from Madeira. In: Proc. Zool. Soc. London 1866 (S. 311).

*) Jordan \& Gilbert, 1882 - Synopsis of the fishes of North America. In: Bull. U.S. Nat. Mus., 5, Pt. 3, Nr. 16 (S. 458).

*) Jordan \& Evermann, 1896/99 - The fishes of North and middle America, 5, Pt. 1 (S. 837).

Joubin, 1932 - s. bei Le Gall.

Koefoed, E., 1927 - Fishes from the sea bottom. In: Rep. Sci. Res. "Michael Sars" North Atl. Deap-Sea Exp., 1910, 4, Pt. 1 (S. 125).

Köhler, R., 1896 - Résultates scientifiques de la campagne du "Caudan“ dans le Golf de Gascogne. In: Ann. Univ. de Lyon, 1896 (S. 484).

Kotthaus, A., 1950 - Merkwürdige Fische von der südisländischen Küste. In: Fischereiwelt, 1950 (S. 12-13).

*) Lowe, R. T., 1843 - A History of the fishes of Madeira, 1843 (S. 54).

L ücke, F., 1947 - Der Seefisch als Nahrungsmittel. In: Fischwoche, 2, Heft 19/22 (S. 130-132).

L ütken, Chr., 1880 - Formforandringer hos Fiske. In: Spolia atlantica, 1880 (S. 428).

Moreau, E., 1881 - Histoire naturelle des poissons de la France, 2 (S. 322-326).

Murray \& Hjort, 1912 - The depths of the ocean, London 1912.

Norman, J.R., 1939 - Sci. Rep. on the "John Murray“ Exp., 7, Pt. 1, Fishes (S. 55).

*) Owen, R, 1866 - Anatomy of vertebrates, Pt. 1.

Rauther, M., 1940 - Anatomie, Physiologie und Entw.-Geschichte der Fische. In: Bronns, Klassen und Ordnungen des Tierreichs, 6, I. Abt., 2. Buch, Teil 1, 1. Hälfte.

Schultz \& Stern, 1948 - The ways of fishes, New York.

Smith, J.L., 1950 - The sea fishes of Southern Africa.

Soljan, T., 1948 - Ribe Jadrana. In: Fauna i Flora Jadrana, 1.

*) Starks, E. Ch., 1904 - The osteology of some berycoid fishes. In: Proc. U.S. Nat. Mus., 1904, 27 (S. 601-619).

Steindachner \& Döderlein, 1883 - Beitr. z. Kenntn. der Fische Japans. In: Denkschr. K. Akad. Wiss., Math.-naturw. Classe, 47 (S. 218).

Supino, F., 1902 - Ricerche sul cranio dei Teleostei, Tl, 4, Pomatomus, Hoplostethus. In: Ric. Lab. Anat. Roma, 1902 (S. 217-231).

Vaillant, 1888 - Exped. Sci. du "Traveilleur ${ }^{\circ}$ et du "Talisman" pendant les années 1880-1883, Poissons. Herausgegeb. v. A. Milne Edwards.

Weber \& Beaufort, 1929 - The fishes of the Indio-Australian archipelagos, 5 (S. 216). Wille, O., 1949 - Der Fisch, 3.

Wüst, G., 1951 - Über die Fernwirkung antarktischer und nordatlantischer Wassermassen in den Tiefen des Weltmeeres. In: Naturw. Rundschau, 4, Heft 3 (S. 97-108) 